\title{
Synaptic Circuit Organization of Motor Corticothalamic Neurons
}

\author{
Naoki Yamawaki and Gordon M.G. Shepherd \\ Department of Physiology, Feinberg School of Medicine, Northwestern University, Chicago, Illinois 60611
}

Corticothalamic (CT) neurons in layer 6 constitute a large but enigmatic class of cortical projection neurons. How they are integrated into intracortical and thalamo-cortico-thalamic circuits is incompletely understood, especially outside of sensory cortex. Here, we investigated CT circuits in mouse forelimb motor cortex (M1) using multiple circuit-analysis methods. Stimulating and recording from CT, intratelencephalic (IT), and pyramidal tract (PT) projection neurons, we found strong CT $\leftrightarrow$ CT and CT $\leftrightarrow$ IT connections; however, $\mathrm{CT} \rightarrow$ IT connections were limited to IT neurons in layer 6, not 5B. There was strikingly little CT $\leftrightarrow$ PT excitatory connectivity. Disynaptic inhibition systematically accompanied excitation in these pathways, scaling with the amplitude of excitation according to both presynaptic (class-specific) and postsynaptic (cell-by-cell) factors. In particular, CT neurons evoked proportionally more inhibition relative to excitation (I/E ratio) than IT neurons. Furthermore, the amplitude of inhibition was tuned to match the amount of excitation at the level of individual neurons; in the extreme, neurons receiving no excitation received no inhibition either. Extending these studies to dissect the connectivity between cortex and thalamus, we found that M1-CT neurons and thalamocortical neurons in the ventrolateral (VL) nucleus were remarkably unconnected in either direction. Instead, VL axons in the cortex excited both IT and PT neurons, and CT axons in the thalamus excited other thalamic neurons, including those in the posterior nucleus, which additionally received PT excitation. These findings, which contrast in several ways with previous observations in sensory areas, illuminate the basic circuit organization of CT neurons within M1 and between M1 and thalamus.

Key words: cortex; corticothalamic; disynaptic inhibition; layer 6; projection neuron; VA/VL

\section{Introduction}

Corticothalamic neurons in layer 6 (CT neurons), distinguished by their axonal projections to thalamus including the reticular nucleus (RTN), are one of three major classes of cortical projection neurons, along with intratelencephalic (IT) neurons (projecting to cortex/striatum; e.g., corticocallosal neurons) and pyramidal tract (PT) neurons (with subcerebral projections; e.g., corticospinal neurons) (for review, see Shepherd, 2013). Determining the interconnectivity of CT neurons with IT and PT neurons is an important step toward understanding the circuit-level substrates of information processing within the cortex. Previous studies of CT neurons' circuits have mostly been conducted in sensory cortical areas (for review, see Thomson, 2010). Much less is known about CT circuits in agranular areas. In mouse motor cortex (M1), recent studies have focused on IT and PT neurons and their connectivity within and across projection classes and

\footnotetext{
Received Sept. 27, 2014; revised Nov. 24, 2014; accepted Dec. 27, 2014.

Author contributions: N.Y. and G.M.G.S. designed research; N.Y. performed research;N.Y. analyzed data; N.Y. and G.M.G.S. wrote the paper.

This work was supported by the National Institute of Neurological Disorders and Stroke, National Institutes of Health (Grant NS061963). We thank K. Borges, B. Suter, L. Sybert, and A. Tanimura for advice and assistance; C. Gerfen, J. Surmeier, and S. Ulrich for mice; and K. Borges, K. Harris, and K. Svoboda for discussions and valuable input. The authors declare no competing financial interests.

Correspondence should be addressed to Gordon M.G. Shepherd, MD, PhD, Department of Physiology, Feinberg School of Medicine, Northwestern University, Morton 5-660, 303 E. Chicago Ave., Chicago, IL 60611. E-mail: g-shepherd@northwestern.edu.

DOI:10.1523/JNEUROSCI.4023-14.2015

Copyright $\odot 2015$ the authors $\quad 0270-6474 / 15 / 352293-15 \$ 15.00 / 0$
}

sublayers (Anderson et al., 2010; Kiritani et al., 2012). How, and even whether, M1-CT neurons interconnect with IT and PT neurons remains poorly understood.

Similar uncertainty pertains to the interconnectivity of CT neurons with thalamic neurons. In primary sensory cortex, CT neurons project back to the same first-order thalamic nucleus providing ascending driver-type thalamocortical (TC) afferents, whereas PT neurons project instead to higher-order thalamic nuclei (Thomson, 2010; Sherman, 2012). In motor areas, a similar pattern is thought to pertain, but has received less experimental evaluation. In the thalamus $\rightarrow \mathrm{M} 1$ direction, the connectivity of TC axons from motor thalamus onto different types of M1 neurons has begun to be addressed electrophysiologically (Hooks et al., 2013), but the thalamo-recipience of CT neurons has not been fully resolved. In the $\mathrm{M} 1 \rightarrow$ thalamus direction, these projections have been described anatomically (Terashima et al., 1987; Puelles et al., 2012; Hooks et al., 2013; Oh et al., 2014), but excitatory connectivity has not been assessed electrophysiologically, in contrast to sensory CT projections (Landisman and Connors, 2007; Cruikshank et al., 2010; Olsen et al., 2012).

To address these open questions about CT neuron circuits, we investigated the synaptic connectivity whereby CT neurons in M1 are incorporated into intracortical circuits with IT and PT neurons and into thalamo-cortico-thalamic circuits with thalamic neurons. For this, optogenetic-electrophysiological approaches were used to selectively photostimulate and record from identified neurons. Our findings illuminate the synaptic circuit orga- 
nization of CT neurons and suggest a novel view on how information is processed in intracortical and thalamo-corticothalamic circuits in the motor system.

\section{Materials and Methods}

Study approval and mice. Studies were approved by the Northwestern University Animal Care and Use Committee. Mice (C57BL/6, female and male) were bred in-house or purchased from The Jackson Laboratory.

Labeling projection neurons with tracers and ChR2. Most experiments followed previously published methods (Kiritani et al., 2012) to label, photostimulate, and record from motor cortex (M1) projection neurons. Briefly, for retrograde labeling, mice were injected at postnatal day 23-25 with fluorescent tracers into the ipsilateral ventrolateral (VL) nucleus, the contralateral spinal cord (C2 level), or the contralateral M1 or dorsolateral striatum to label, respectively, representative types of CT, PT (i.e., corticospinal), and IT (i.e., corticocallosal) projection neurons. Tracers used included latex microspheres (red Retrobeads; Lumafluor) or cholera toxin subunit B conjugated to Alexa Fluor (CTB647; Life Technologies). Similarly, and during the same procedure, deletionmutant rabies virus (RV) carrying channelrhodopsin-2 (ChR2) and the fluorescent protein Venus (RV-ChR2) (Osakada et al., 2011; Kiritani et al., 2012) was injected into one of these same targets, resulting in ChR2expressing CT, IT, or PT projection neurons. Therefore, in each animal, two types of projection neurons were labeled by retrograde tracers (of different colors) and one was labeled by RV-ChR2. Coronal brain slices $(0.3 \mathrm{~mm})$ were prepared 5-9 d later. Recordings were targeted to tracerlabeled ChR2-negative neurons unless otherwise stated.

In some experiments, we injected adeno-associated virus (AAV) carrying ChR2 (AAV-flex-ChR2; AAV1.CAGGS.Flex.ChR2-tdTomato. WPRE.SV40, AV-1-18917P, University of Pennsylvania Vector Core) into M1 in Ntsr1-Cre mice (line GN220, MMRRC) (Gong et al., 2007) to selectively transfect CT neurons. To minimize damage to the cortex, the glass injection pipette was pulled to a fine tip and beveled to a sharp edge (Micro Grinder EG-400; Narishige) and slowly advanced into M1 at an angle of $10^{\circ}$ off the vertical axis of the cortex. Injections were made slowly (3 min) at 2 depths ( 0.8 and $1.2 \mathrm{~mm}$ from pia, $\sim 20 \mathrm{~nL}$ per injection). Different classes of cortical projection neurons were labeled with different-colored tracers (green microspheres or CTB647) in the same mice, as described above. In other experiments, these mice or Sim1_KJ18-Cre mice (Gerfen et al., 2013) were used to examine CT or PT connections, respectively, to TC neurons that project back to M1 (M1p-TC neurons). For this, M1 was injected first with AAV-flex-ChR2 (for Sim1_KJ18: 0.8 and $0.5 \mathrm{~mm}$ from pia, $\sim 20 \mathrm{~nL}$ per injection) and then with the retrograde tracer. Coronal brain slices $(0.3 \mathrm{~mm})$ were prepared 21-28 d later and recordings were targeted to cortical projection neurons labeled with retrograde tracers.

For experiments examining TC input to $\mathrm{M} 1$ projection neurons, AAVChR2 (AAV1.CAG.ChR2-Venus.WPRE.SV40, AV-1-PV2126, University of Pennsylvania Vector Core) was used. The use of small injection volumes $(\sim 25 \mathrm{~nL})$ and stereotaxic targeting of VL resulted in thalamic labeling that was mostly restricted to VL. Some additional labeling in surrounding regions such as the posterior (PO) nucleus, which is located posterior to VL and is a source of input to upper-layer neurons in vibrissal M1 (Hooks et al., 2013; Hunnicutt et al., 2014), may have occurred, but the highly specific axon labeling and connectivity patterns we report in this study strongly suggest that this did not represent a significant confound (see also Results). Furthermore, the ventral anterior nucleus, located rostral to VL and known to be functionally and anatomically distinct from VL in rats (Kuramoto et al., 2009), is less distinct from VL in mouse (Puelles et al., 2012) and may therefore have been colabeled to a minor extent. Injection of retrograde tracer of one color was stereotaxically targeted to the same VL coordinates after virus injection to label CT neurons, and retrograde tracer of another color was injected into either spinal cord or contralateral M1 to label PT or IT projection neurons, respectively. Coronal brain slices $(0.3 \mathrm{~mm})$ were prepared $21-28 \mathrm{~d}$ later and recordings were targeted to cortical projection neurons labeled with retrograde tracers.

Electrophysiology and photostimulation. Pipettes contained potassiumbased internal solution for laser scanning photostimulation (LSPS) and
AAV-ChR2 experiments, in which inhibitory inputs were not sampled, and cesium-based internal solution for RV-ChR2 (and some Ntsr1ChR2) experiments, in which they were (composition, in mM: 128 potassium or cesium methanesulfonate, 10 HEPES, 10 phosphocreatine, 4 $\mathrm{MgCl}_{2}$, 4 ATP, 0.4 GTP, 3 ascorbate, 1 EGTA, 1 QX-314, pH 7.25, 290 $295 \mathrm{mOsm})$. A command potential of -70 or $0 \mathrm{mV}$ was applied to isolate either excitatory (glutamatergic) or inhibitory (GABAergic) postsynaptic currents (EPSC or IPSC), respectively (Wood et al., 2009). In all experiments, CPP $(5 \mu \mathrm{M})$ was added to bath solution to block NMDA-receptormediated currents, thereby dampening excitability. LSPS experiments were performed at $21^{\circ} \mathrm{C}$ and all other recordings were at $32^{\circ} \mathrm{C}$.

Optogenetic photostimulation experiments were conducted in either "CRACM" (for "ChR2-assisted circuit mapping") (Petreanu et al., 2007) or "sCRACM" (for "subcellular CRACM") (Petreanu et al., 2009) mode. In sCRACM mode, the sodium channel blocker tetrodotoxin (TTX, 1 $\mu \mathrm{M})$ and the potassium channel blocker 4-aminopyridine (4-AP, 100 $\mu \mathrm{M})$ are added to the bath solution to isolate monosynaptic EPSCs (only). In CRACM mode, omission of these reagents permits both EPSCs and IPSCs to be sampled. Although in CRACM mode there is a theoretical possibility of higher-order (disynaptic and polysynaptic) EPSCs, the responses we observed are likely to represent primarily monosynaptic EPSCs, because: (1) excitation was generally dampened (by blocking NMDA receptors), (2) EPSC waveforms lacked longer-latency components, and (3) the highly specific connectivity patterns appear incompatible with nonspecific "spillover" excitation that might arise from polysynaptic activity. Prior studies have shown good correspondence between connectivity patterns measured with RV-ChR2 and paired recordings (Kiritani et al., 2012). As in previous studies (Apicella et al., 2012; Kiritani et al., 2012), CRACM mode was used for all intracortical connectivity experiments involving RV-ChR2, both for the practical reason that photoexcitability levels were too low for reliable detection under sCRACM conditions and because this mode allowed IPSCs to be sampled along with EPSCs. CRACM mode was also used for all experiments involving AAV-ChR2 to examine M1 excitatory inputs to thalamic neurons because polysynaptic excitation was not a concern (due to the general absence of feedforward excitatory microcircuits within rodent thalamus; Lee et al., 2010) and because this allowed spiking responses to be evaluated in a subset of cells. In contrast, in experiments involving AAV-ChR2 to examine TC inputs to M1 neurons, it was critical to use sCRACM mode to isolate monosynaptic inputs (Petreanu et al., 2009) because polysynaptic excitation was a theoretical concern in light of the intracortical connectivity patterns identified in the RV-ChR2 experiments (e.g., $\mathrm{TC} \rightarrow \mathrm{IT} \rightarrow \mathrm{CT}$ ).

Wide-field ChR2 photostimulation was performed using a $4 \times$ objective lens (UPlanSApo, numerical aperture 0.16; Olympus) focused on the slice, by briefly ( $5 \mathrm{~ms}$ ) gating the output of a blue LED (M470L2; Thorlabs, $1.27 \mathrm{~mW} / \mathrm{mm}^{2}$ intensity at the surface of specimen). Responses to multiple trials were sampled at an interstimulus interval of $30 \mathrm{~s}$. Recordings with series resistance $>40 \mathrm{M} \Omega$ were excluded. Data were acquired using Ephus software (Suter et al., 2010). Signals were amplified with an Axon Multiclamp 700B (Molecular Devices), filtered at $4 \mathrm{kHz}$, and sampled at $10 \mathrm{kHz}$. Traces were analyzed using custom MATLAB (The MathWorks) routines. For each neuron, two or more traces were averaged and the evoked response was computed as the mean current over a poststimulus interval of $50 \mathrm{~ms}$. For connectivity studies among M1 projection neurons, recordings were targeted to pairs of CT and IT or CT and PT neurons in the slice, recorded sequentially in varying order. In some cases, a third neuron was sampled, allowing two pairwise comparisons among the three neurons. The pairs of neurons were always vertically aligned as well as possible (horizontal offset typically close to $0 \mu \mathrm{m}$ and maximally $100 \mu \mathrm{m}$ ) and, for pairs in which both neurons were in layer 6 , the vertical offset was also minimized (i.e., neighboring neurons). For experiments examining TC connections to different projection neurons, we sampled multiple identified neurons at various cortical depths across the deeper layers to obtain a laminar input profile. The slice was positioned at the same location under the $4 \times$ objective for both neurons in each pair. For experiments assessing CT connections to thalamic neurons, in each nucleus of interest we recorded from multiple M1p-TC neurons at a variety of locations 

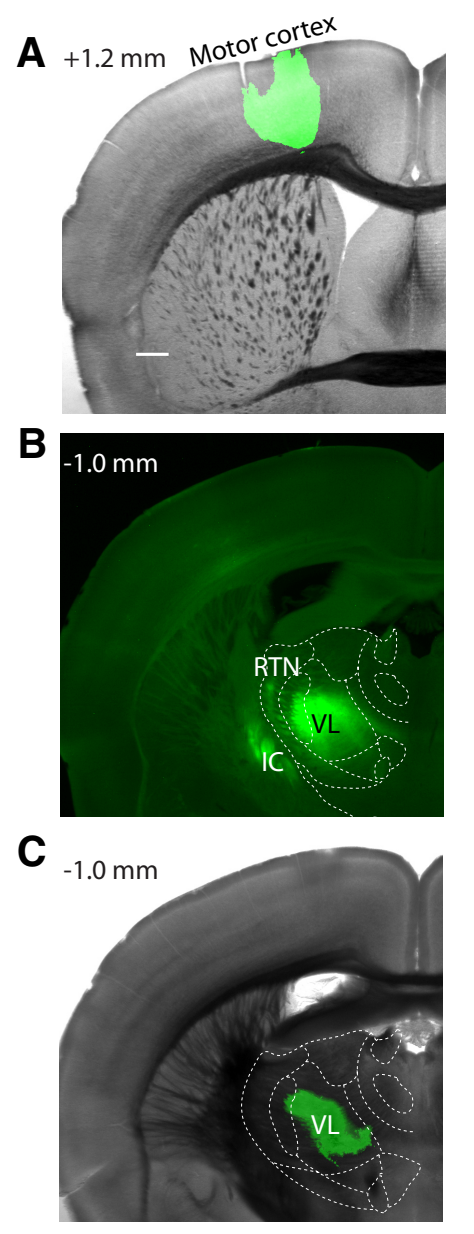
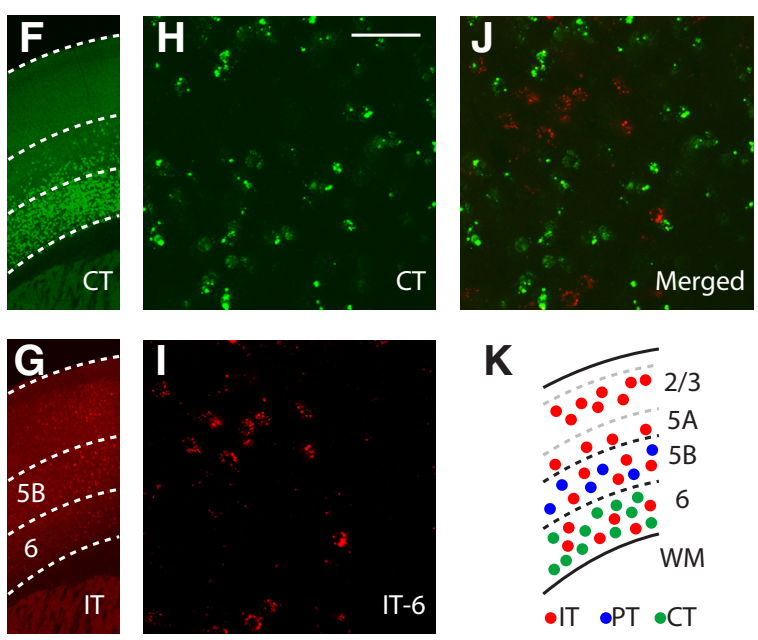

E

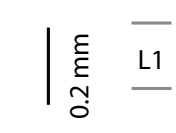

$\mathrm{L} 2 / 3$

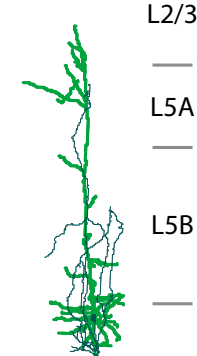

L6

WM

Figure 1. CT neurons are a distinct class of M1 projection neurons intermingled with IT neurons in layer 6 . $A$, Bright-field image of coronal brain slice through $M 1$ with superimposed epifluorescence image showing location of AAV-GFP injection site (green). Scale bar, $0.5 \mathrm{~mm}$. Estimated anterior-posterior distance from bregma is indicated in top left corner. $\boldsymbol{B}$, Epifluorescence image of the resulting labeling pattern of $\mathrm{M} 1$ axons in thalamus. Outlines of thalamic nuclei are based on comparison of bright-field images with reference atlases (Paxinos and Franklin, 2001; Dong, 2008). IC, Internal capsule. C, Bright-field and superimposed epifluorescence image showing the retrograde tracer injection site in VL. D, Confocal image of retrogradely labeled neurons in M1 after tracer injection in VL. WM, White matter. Scale bar, $0.5 \mathrm{~mm}$. $\boldsymbol{E}$, Reconstruction of a retrogradely labeled neuron. Thin lines are axons. Laminar borders are based on Suter et al. (2013). $\boldsymbol{F}$, Retrograde labeling pattern of (T neurons (from D). G, Retrograde labeling pattern of IT neurons distributed in layers $2-6$ (same slice; red tracer injected in contralateral M1 to label corticocallosal neurons). $\boldsymbol{H}$, $\boldsymbol{I}$, Higher-magnification confocal image of labeled CT neurons $(\boldsymbol{H})$ and IT neurons $(\boldsymbol{I})$ in layer 6 of M1. Scale bar, $50 \mu \mathrm{m}$. $\boldsymbol{J}$, Merged image. $\boldsymbol{K}$, Schematic representation of laminar distributions of CT, IT, and PT neurons in M1.

within the nucleus. This was usually achieved by sampling neurons located laterally, medially, and in the center of the region containing M1p-TC neurons, with additional sampling along the dorsoventral axis if possible.

LSPS by glutamate uncaging was performed as described previously for mapping intracortical connectivity in mouse motor cortex (Weiler et al., 2008; Anderson et al., 2010; Shepherd, 2012; Hooks et al., 2013). Recording conditions (including $21^{\circ} \mathrm{C}$ bath solution containing the NMDA-receptor antagonist CPP and increased concentrations of $\mathrm{Ca}^{2+}$ and $\mathrm{Mg}^{2+}$ ) were designed to dampen neuronal excitability, enabling mapping of compound monosynaptic inputs from hundreds of discrete locations across the slice (for details and additional information about the acquisition, analysis, and interpretation of LSPS data, see Schubert et al., 2001; Shepherd et al., 2003; Weiler et al., 2008; Shepherd, 2012). In these experiments, coronal brain slices $(0.3 \mathrm{~mm})$ were prepared $3-7 \mathrm{~d}$ after in vivo tracer injections and recordings were targeted to cortical projection neurons labeled with retrograde tracers.

Statistical analysis. Group data are presented as mean \pm SEM unless otherwise indicated. Group comparisons were made using nonparametric tests (sign test for median, signed-rank test for mean, and rank-sum test, as indicated), with significance defined as $p<0.05$.

\section{Results}

Retrograde labeling identifies M1-CT neurons projecting to $\mathrm{VL}$

As a starting point, we began by anatomically localizing the M1 projection to thalamus so that we could subsequently target this thalamic region for injections of retrograde tracers and viruses to label M1-CT neurons. Injection of AAV-GFP into the forelimb area of M1 (Fig. 1A) resulted in fluorescent labeling of axons in an anterior thalamic region encompassing the VL nucleus (Fig. 1B). This prominent $\mathrm{M} 1 \rightarrow \mathrm{VL}$ projection was consistent with expectations from prior studies in diverse mammalian species including mice (Tlamsa and Brumberg, 2010; Puelles et al., 2012; Hooks et al., 2013; Oh et al., 2014) and is among the reasons why VL, which receives primarily cerebellar afferents (Puelles et al., 2012), is often considered "motor thalamus." Labeling was also observed in the RTN and other thalamic nuclei that will be addressed later.

Injection of retrograde tracer in VL (Fig. 1C) resulted in fluorescent labeling of a large population of CT neurons in layer 6 of 
A
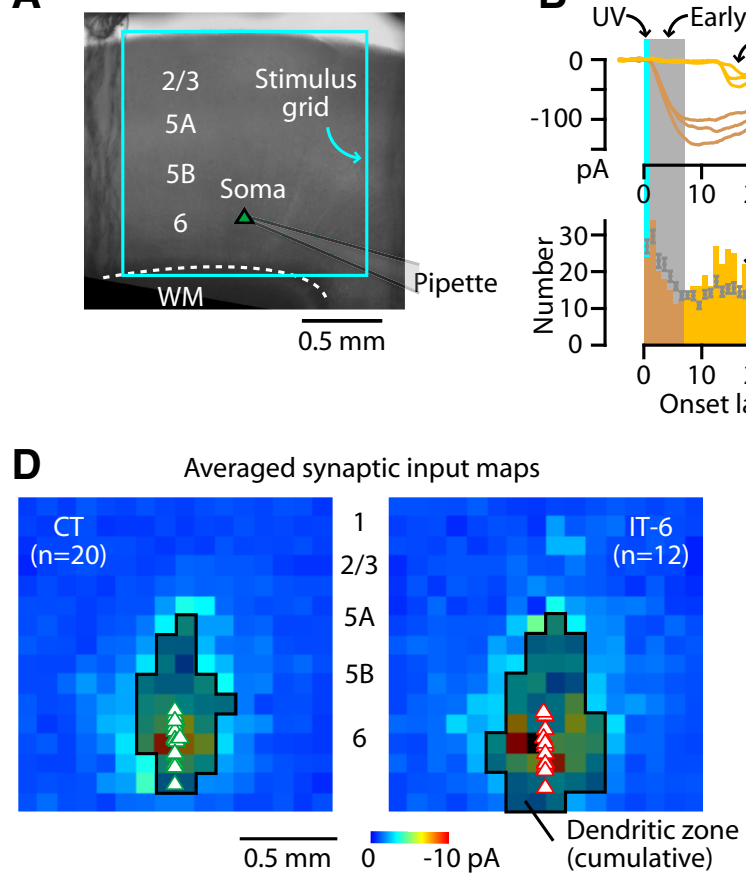

B

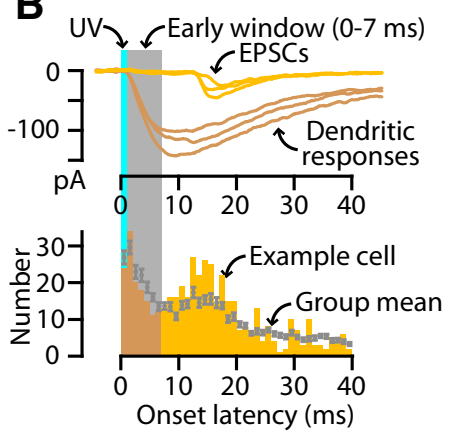

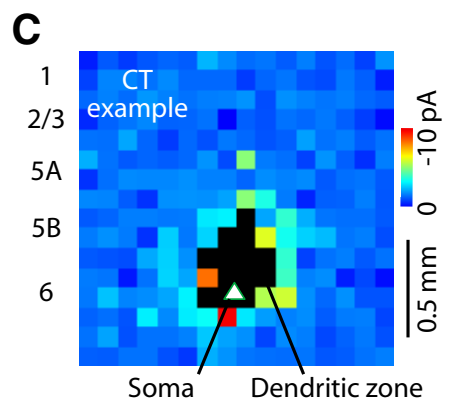

E

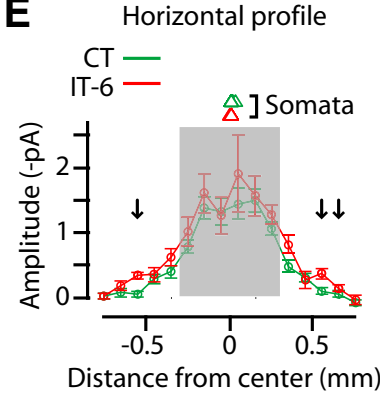

Vertical profile

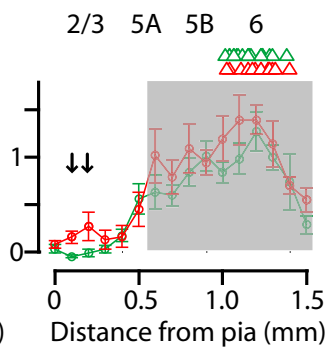

Figure 2. Local excitatory input to M1-CT neurons comes from nearby sites in deeper layers. $A$, Recording configuration for mapping inputs to CT neurons using glutamate uncaging and laser scanning photostimulation. Bright-field image of $\mathrm{M} 1$ viewed with a $4 \times$ objective lens showing orientation of the mapping grid. $\boldsymbol{B}$, Top, Example traces with responses recorded in a CT neuron evoked by focal glutamate uncaging at various sites in the grid. Synaptic inputs (EPSC, yellow traces) arrive with longer latencies than dendritic responses (orange traces). Bottom, Histogram of onset latencies. A 7 ms time window (gray shadow) was used to identify dendritic responses and exclude them from further analysis. C, Example of a (T neuron's excitatory synaptic input map. Pixels represent the mean current amplitude in the poststimulus time interval of $7-50 \mathrm{~ms}$. Black pixels represent the zone of dendritic responses, identified by latency analysis (see $\boldsymbol{B}$ ). $\boldsymbol{D}$, Average synaptic input maps of (T neurons and IT-6 neurons. Shaded pixels (outlined in black) indicate sites where dendritic responses were observed in any of the neurons. $E$, Horizontal (left) and vertical (right, with layers indicated above plot) profiles of (T (green) and IT-6 (red) synaptic input maps. Arrows indicate significant CT-IT differences (rank-sum test, $p<0.05$ ). Gray shading indicates regions with dendritic responses.

M1 (Fig. 1D). Scattered PT neurons in layer 5B were also labeled, consistent with observations that thalamus is among the many possible subcortical targets of PT axons (Hattox and Nelson, 2007; Kita and Kita, 2012). Although the border of layer 6 with layer $5 \mathrm{~B}$ is indistinct under bright-field optics, it can be defined based on the lower border of the laminar pattern of PT labeling, which in mouse forelimb M1 occurs at a normalized cortical depth of $\sim 0.75$ (pia $=0$, white matter $=1$; Suter et al., 2013). Consistent with this, the transition between the high-density labeling (i.e., in layer 6) and low-density labeling (i.e., in layer 5B) observed in slices after retrograde tracer injection in VL coincided with this expected location of the layer $5 \mathrm{~B} / 6$ border.

Reconstructions of retrogradely labeled CT neurons (Fig. 1E) showed apical dendrites that extended to upper layer 5A, similar to CT neurons in rodent somatosensory cortex (Zhang and Deschênes, 1997; Kumar and Ohana, 2008; Kim et al., 2014). In sensory cortex, CT neurons are intermingled with but distinct from IT neurons in layer 6 (Thomson, 2010). To assess this in $\mathrm{M} 1$, we coinjected retrograde tracers of different colors into VL to label CT neurons and into contralateral M1 to label IT neurons. Zero of $268 \mathrm{CT}$ neurons counted were double-labeled (Fig. $1 F-J$ ), indicating that CT and IT neurons are nonoverlapping neuronal populations in M1. PT and IT neurons are also nonoverlapping populations (for review, see Shepherd, 2013). Therefore, in M1, layer 6 contains CT and IT neurons, layer 5B contains PT and IT neurons, and upper layers contain only IT neurons (Fig. $1 K$ ). In the experiments in M1 slices that follow, recordings were in all cases targeted to CT, IT, or PT neurons identified on the basis of retrograde labeling.
Local excitatory input to M1-CT neurons comes from nearby sites in deeper layers

We examined the local sources of excitatory input to M1-CT neurons by surveying these connections using glutamate uncaging and LSPS. We targeted whole-cell recordings to retrogradely labeled CT and, for comparison, to IT neurons (corticocallosal neurons, labeled by tracer injection in contralateral M1) in layer 6 (IT-6), and oriented the stimulus grid to span all layers and an area of $2.25 \mathrm{~mm}^{2}$ around the neuron (Fig. $2 A$ ). In responses evoked by flashing the laser beam at each of 256 sites across this region, synaptic inputs (from photostimulated presynaptic neurons) were readily distinguished from direct stimulation of the recorded neuron's dendrites based on latency (Fig. 2B). Traces were analyzed to construct a synaptic input map for each neuron (Fig. 2C), and the maps of multiple CT neurons and IT-6 neurons were averaged for each group (Fig. 2D). Consistent with prior findings for unlabeled layer 6 neurons in M1 (Weiler et al., 2008; Hooks et al., 2011), excitatory inputs to both CT and IT-6 neurons arose primarily from nearby sites in deeper layers $(5 \mathrm{~A}, 5 \mathrm{~B}$, and 6). The IT-6 neurons received inputs over a slightly broader horizontal extent (Fig. 2E, left) and, unlike CT neurons, received a small amount of input from layer $2 / 3$ (Fig. 2E, right); however, although significantly different, the response amplitudes at these locations were small on average.

Because the deeper layers contain a mix of CT, IT, and PT neurons (see above and Fig. $1 K$ ), we next turned to optogenetic methods to evaluate the cell types that could be involved in these connections. 
A
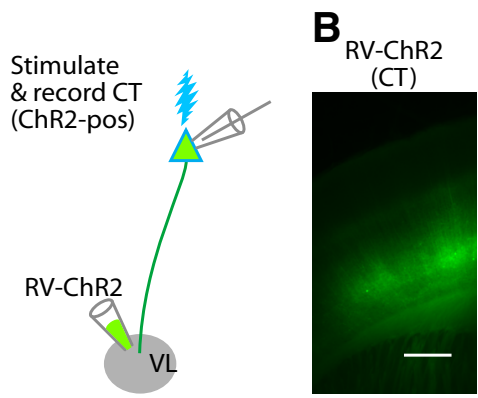

D

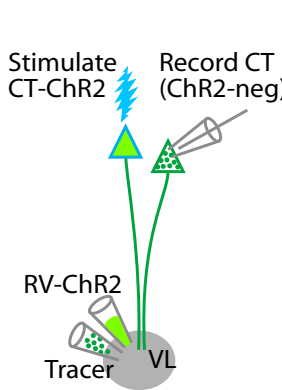

E

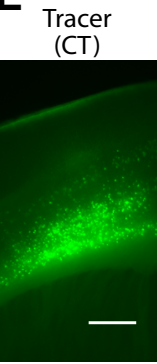

G

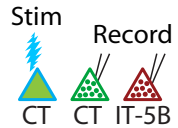

(RV)

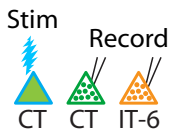

(RV)

H
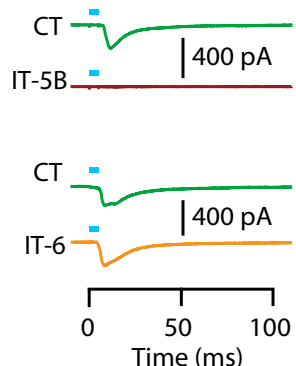

K

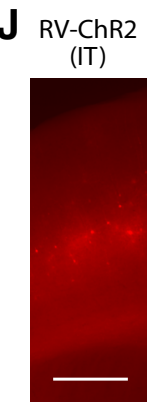

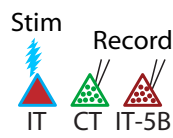

(RV)

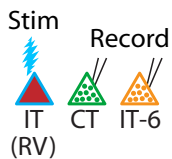

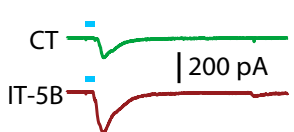

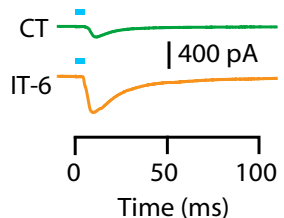

C

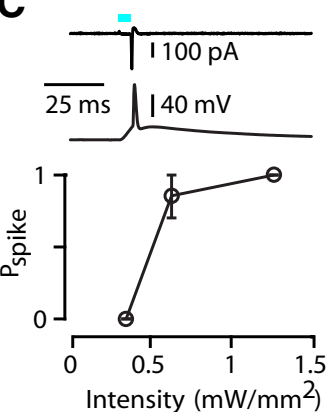

$\mathbf{F}$
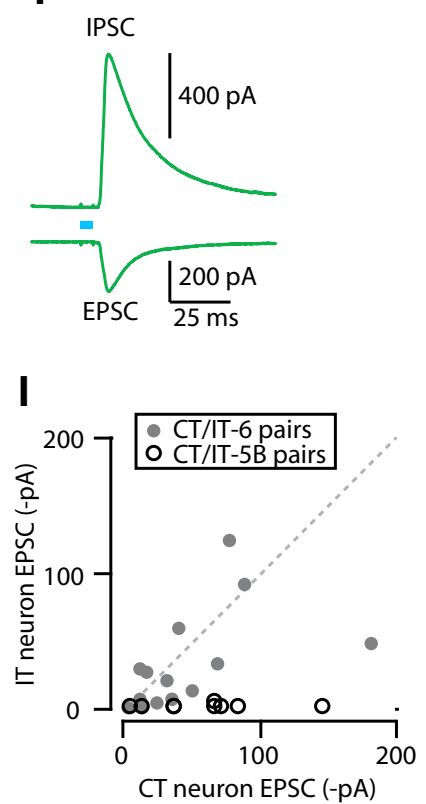

$\mathbf{L}$

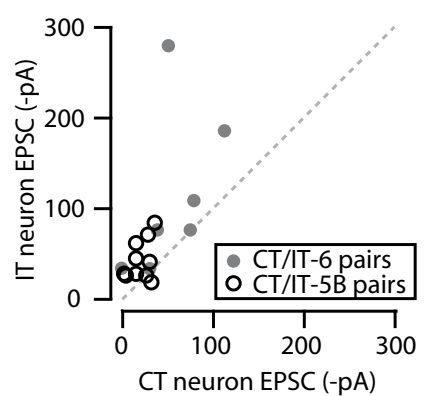

Figure 3. CT and IT neurons are interconnected in a partially layer-specific manner. $\boldsymbol{A}$, Injection of RV-ChR2 into VL transfects CT neurons for optogenetic photostimulation. $\boldsymbol{B}$, Epifluorescence image of RV-ChR2-transfected CT neurons in M1. Scale bar, $0.4 \mathrm{~mm}$. C, Example traces of photo-evoked spikes recorded from a ChR2-expressing CT neuron, first in cell-attached (upper) and then whole-cell (middle) configuration. Bottom, Plot of photo-evoked spike probability $\left(P_{\text {spike }}\right)$ as a function of light intensity. Stimulation width was $5 \mathrm{~ms}$. D, Injection of both RV-ChR2 and inert retrograde tracer into VL for synaptic connectivity analysis. For simplicity, the double-labeling of many CT neurons is omitted. $\boldsymbol{E}$, Epifluorescence image of CT neurons labeled with retrograde tracer (CTB647). F, Example traces of a photo-evoked EPSC and IPSC recorded from identified CT neuron. $\mathbf{G}$, Schematic of stimulation/recording configuration. $\boldsymbol{H}$, Example traces of EPSCs recorded from CT and IT-5B (top) and CT and IT- 6 (bottom) neurons upon photostimulation of CT neurons. I, Pairwise comparison of photo-evoked EPSC between CT and IT-5B (open circles) or CT and IT- 6 neurons (filled circles). Line indicates unity slope. $J$, Epifluorescence image (left) and schematic of stimulation/recording configuration (right). Scale bar, $0.5 \mathrm{~mm}$. $\boldsymbol{K}$, Example traces of EPSCs recorded from CT and IT-5B (top) and CT and IT-6 (bottom) neurons upon photostimulation of IT neurons. $L$, IT input to CT versus IT neurons, as in $I$.

\section{Photostimulation of CT neurons using deletion-mutant RV encoding ChR2}

To investigate which projection classes provide the presynaptic intracortical excitatory input to CT neurons, we used an ap- proach based on deletion-mutant rabies virus encoding $\mathrm{ChR} 2$ and Venus (RVChR2), a tool for retrogradely transfecting cortical projection neurons for optogenetic photostimulation experiments (Osakada et al., 2011; Kiritani et al., 2012). Consistent with prior RV-ChR2 experiments with PT neurons (Kiritani et al., 2012), control experiments showed that injection of RV-ChR2 into VL resulted in numerous retrogradely transfected (i.e., ChR2-expressing) CT neurons in M1 slices (Fig. 3A,B). Electrophysiological recordings from these transfected CT neurons indicated robust and reliable photoexcitability, with a single action potential evoked per photostimulus (6/6 neurons at an LED intensity of $1.27 \mathrm{~mW} /$ $\mathrm{mm}^{2}$; Fig. 3C).

When VL was injected with both RVChR2 and an inert and spectrally distinct retrograde tracer (e.g., CTB647; Fig. 3D), subsequently prepared M1 brain slices contained a subset of CT neurons labeled only with the tracer (Fig. $3 D, E$ ). Wholecell recordings from these untransfected, tracer-positive/ChR2-negative CT neurons showed robust synaptic responses after photostimulation of the transfected CT neurons (Fig. $3 F$ ). In these intracorti$\mathrm{cal} / \mathrm{RV}$ experiments, we used experimental conditions designed to allow sampling of both EPSCs and IPSCs (see Materials and Methods). Recordings at $-70 \mathrm{mV}$ command potential showed robust photoevoked EPSCs, indicating strong recurrent $(\mathrm{CT} \rightarrow \mathrm{CT}$ ) connectivity (Fig. $3 F$ ). Recordings at $0 \mathrm{mV}$ showed robust photoevoked IPSCs, indicating strong disynaptic inhibition $(\mathrm{CT} \rightarrow$ interneuron $\rightarrow \mathrm{CT}$; Fig. 3F). The onset latencies of IPSCs lagged those of EPSCs by $1.6 \pm 0.4 \mathrm{~ms}$ (mean \pm SEM, $n=25 \mathrm{CT} \rightarrow$ CT recordings). Application of TTX abolished both the excitatory and inhibitory responses ( $n=2$ neurons), confirming their synaptic basis.

Scattered PT neurons were also labeled by RV-ChR2 after VL injections, as seen for retrograde tracer injection (Fig. 1E). In this experiment, this represents a potential confound, because a component of the photo-evoked responses could arise from photostimulated PT rather than CT neurons. However, as shown below, this was unlikely due to the large asymmetries in the observed connectivity patterns. Another potential confound was the possibility of labeling of thalamic neurons after RV-ChR2 injection in VL. However, we routinely inspected thalamic slices for such labeling and found this to be generally absent (maximally six labeled VL neurons observed in one animal); moreover, the pattern of responses observed here for CT connec- 
tions to $\mathrm{M} 1$ projection neurons (strong $\mathrm{CT} \rightarrow \mathrm{CT}$, little or no $\mathrm{CT} \rightarrow \mathrm{PT}$ or $\mathrm{CT} \rightarrow \mathrm{IT}-5 \mathrm{~B}$ ) is essentially opposite to that observed for VL connections to the same classes of neurons, as will be discussed later. An additional consideration is the possibility that, by only targeting VL for injections, M1-CT neurons projecting to other thalamic nuclei would not be labeled; however, recent anatomical data indicate that VL-projecting CT neurons in mouse M1 arborize extensively in the thalamus, with branches to multiple ipsilateral and contralateral thalamic nuclei including VL, ventromedial nucleus (VM), PO, and RTN (Watakabe et al., 2014), suggesting that this is unlikely to be the case.

With this approach, we systematically evaluated the presynaptic and postsynaptic connectivity of CT neurons with IT and PT neurons: on the presynaptic side, we used retrograde transfection with RV-ChR2 for selectively photostimulating CT, IT, or PT neurons; on the postsynaptic side, we used retrograde labeling with fluorescent tracers to determine the relative strength of photo-evoked synaptic responses in identified projection neurons, comparing CT with either IT or PT neurons in a pairwise manner for neurons recorded sequentially in the same slice.

\section{CT and IT neurons are interconnected in a partially layer- specific manner}

We used RV-ChR2 to assess the output of CT neurons to other CT neurons compared with IT neurons, with the following labeling paradigm. In the same animals, we injected VL with RVChR2, VL with retrograde tracer, and contralateral M1 with a spectrally distinct retrograde tracer, enabling us to record from identified CT and IT neurons for pairwise comparison of their postsynaptic responses upon photostimulation of the presynaptic ChR2-expressing CT neurons (Fig. 3G). This approach furthermore allowed us to compare responses of CT neurons to those of IT neurons in the same (layer 6) or adjacent (layer 5B) layers.

Pairwise comparison of CT versus IT response amplitudes indicated layer-dependent $\mathrm{CT} \rightarrow \mathrm{IT}$ connectivity (Fig. $3 \mathrm{H}, \mathrm{I}$ ): overall, CT connections to IT-5B neurons were only $5 \%$ as strong as those to CT neurons (median ratio of $\mathrm{CT} \rightarrow \mathrm{IT}-5 \mathrm{~B} / \mathrm{CT} \rightarrow \mathrm{CT}$ EPSC amplitude: $0.05 ; p=0.002$, sign test, 10 pairs, 3 animals, 5 slices), but those to IT- 6 and CT neurons were similar (median ratio of $\mathrm{CT} \rightarrow \mathrm{IT}-6 / \mathrm{CT} \rightarrow \mathrm{CT}: 0.58 ; p=0.42$, sign test, 14 pairs, 7 animals, 8 slices). The issue of a possible PT component to the observed IT responses (described above) is negligible in this case because $\mathrm{PT} \rightarrow$ IT connections are weak-to-absent in mouse M1 (Kiritani et al., 2012). In addition, the theoretical possibility that disynaptic CT $\rightarrow$ IT-6 $\rightarrow$ CT activity contributed to the observed responses was unlikely because the experimental conditions favored monosynaptic excitatory responses (see Materials and Methods); consistent with this, EPSCs were monophasic with short onset latencies $(\mathrm{CT} \rightarrow \mathrm{CT}$ and $\mathrm{CT} \rightarrow$ IT-6: $5.5 \pm 0.4$ vs $4.9 \pm$ $0.3 \mathrm{~ms}$, mean \pm SEM; $p=0.19$, signed-rank test, 14 pairs). We also considered the possibility that the lack of CT $\rightarrow$ IT-5B connectivity is a false-positive arising because a hypothetical subclass of CT neurons connecting to IT-5B neurons is either not infected by RV or fails to express ChR2. However, this seems unlikely because of the general efficiency of RV for both infection and transgene expression in mammalian neurons (Wickersham et al., 2007; Wickersham et al., 2010; Osakada et al., 2011; Ginger et al., 2013); furthermore, connectivity patterns observed with RVChR2 have previously been confirmed with paired recordings (Kiritani et al., 2012).

We then assessed connectivity in the reverse direction, from IT to CT and IT neurons. Here, we injected RV-ChR2 into con- tralateral dorsolateral striatum (instead of contralateral M1) to transfect presynaptic IT neurons primarily in layer $5 \mathrm{~B}$, with additional labeling in layers 5A but only sparse labeling in layer $2 / 3$ and 6 (Anderson et al., 2010; Kiritani et al., 2012; Fig. 3J). To label IT neurons for IT-6 recordings, we injected tracer in contralateral M1 (as above); for IT-5B recordings, for convenience, we coinjected (with RV-ChR2) the contralateral dorsolateral striatum, an essentially equivalent way to label these deeper-layer corticocallosal neurons (due to their axonal branching patterns; for review, see Shepherd, 2013).

In general, CT, IT-5B, and IT-6 neurons all received excitatory input from IT neurons. IT- 6 neurons received approximately twice as much IT input compared with neighboring CT neurons (median ratio of $\mathrm{IT} \rightarrow \mathrm{CT} / \mathrm{IT} \rightarrow \mathrm{IT}-6: 0.62 ; p=0.02$, sign test, 7 pairs, 3 animals, 5 slices). IT-5B neurons also received more IT input compared with subjacent CT neurons (median ratio of $\mathrm{IT} \rightarrow \mathrm{CT} / \mathrm{IT} \rightarrow$ IT-5B: 0.43 ; 10 pairs, 3 animals, 6 slices; Fig. $3 \mathrm{~L}$ ), although in this case, the difference was statistically weaker $(p=$ 0.11 by sign test of the median ratios; $p=0.02$ by signed-rank test, normalizing the data to the maximum value per pair).

Together, these data indicate a somewhat complex, quasireciprocal pattern of connectivity between these CT and IT classes of projection neurons in M1. The IT neurons (mostly in layer 5B, based on fluorescent labeling patterns) excited CT neurons, although at $\sim 50 \%$ the amplitude of their connections to other IT neurons (in layers 5B and 6). The CT neurons excited IT neurons, but primarily only those intermingled with them in layer 6 , and not those in layer 5B.

\section{CT and PT neurons are mostly not interconnected}

We next assessed the connections from CT neurons to PT neurons, again compared with CT neurons. To identify PT neurons for targeted recordings, a retrograde tracer was injected into the contralateral cervical spinal cord (see Materials and Methods), resulting in labeling of numerous corticospinal neurons (Anderson et al., 2010; Kiritani et al., 2012). Although corticospinal neurons are a paradigmatic subclass of PT neurons, like PT neurons in general, they are multiprojectional and typically have branches to other subcortical/subcerebral regions, including thalamus (Parent and Parent, 2006; Kita and Kita, 2012; Shepherd, 2013; Shepherd, 2014). In brain slices, we made pairwise recordings from a CT neuron (in layer 6) and a PT neuron (in layer $5 \mathrm{~B}$ ). In an initial set of experiments, we used the same RVChR2 method described above to transfect CT neurons; these results will be described briefly below. However, it became important also to use an alternative method of optogenetically labeling and photostimulating CT neurons without the possibility of a contribution from the scattered transfection of PT neurons with the RV-ChR2 method (discussed earlier). We therefore also tested CT $\rightarrow$ PT connectivity using Ntsr1-Cre mice, in which Cre recombinase expression in neocortex is restricted to $\mathrm{CT}$ neurons (see Materials and Methods). In control experiments, we confirmed the photoexcitability of the ChR2-expressing CT neurons after injection of AAV-flex-ChR2 into M1 (Fig. $4 A-C$ ). Photostimuli excited these transfected CT neurons very strongly, with shorter-duration stimuli $(0.2 \mathrm{~ms})$ producing a level of photoexcitability approximately comparable to RV-ChR2-transfected CT neurons (Fig. 4C). This stimulus duration was used in subsequent experiments involving Ntsr1-Cre mice.

Pairwise comparison of response amplitudes suggested that $\mathrm{PT}$ neurons received relatively little excitatory input from CT neurons (median ratio of $\mathrm{CT} \rightarrow \mathrm{PT} / \mathrm{CT} \rightarrow \mathrm{CT}: 0.20 ; p=0.63$, sign test, 4 pairs, 1 animal, 2 slices for RV-ChR2; and $0.04 ; p=0.01$, 
A

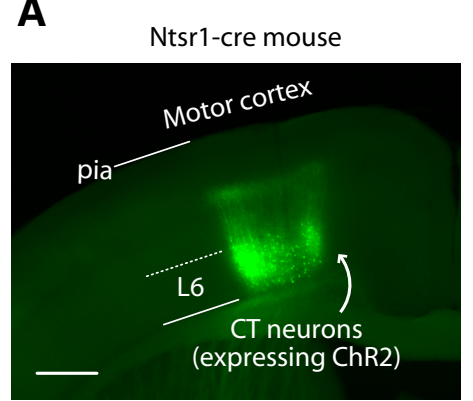

B

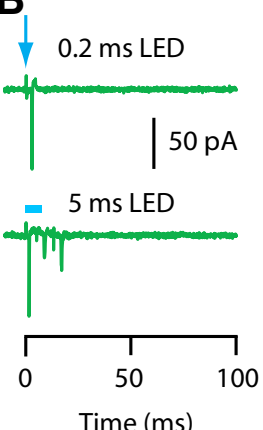

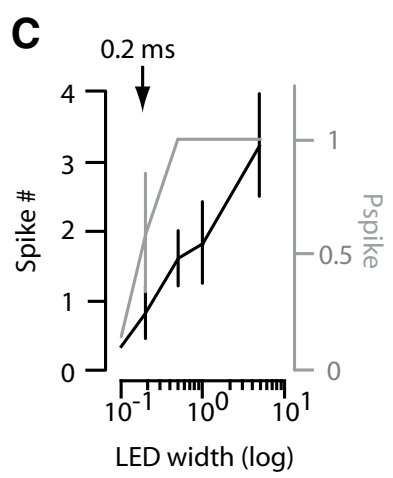

$\mathbf{F}$
D
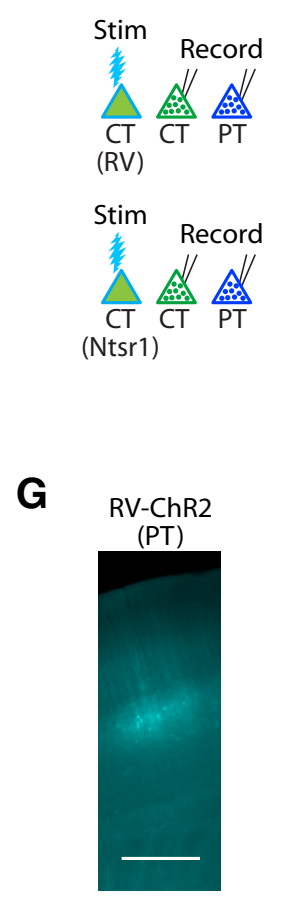

E
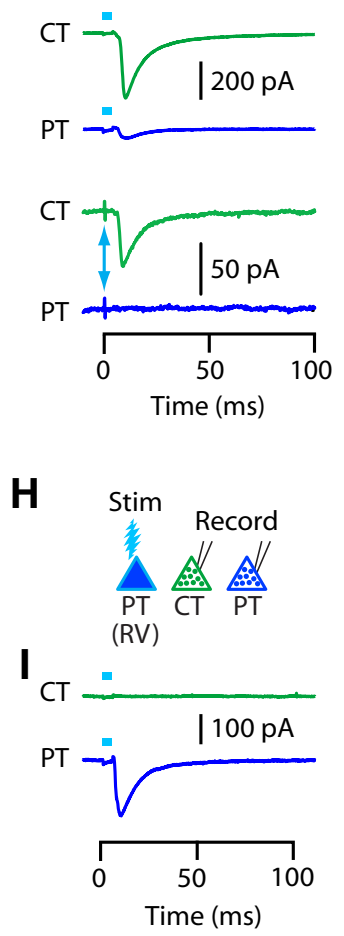

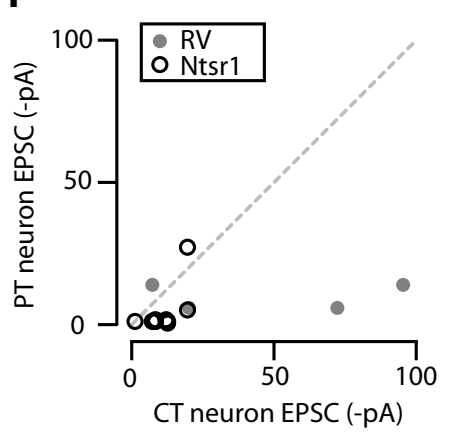

J

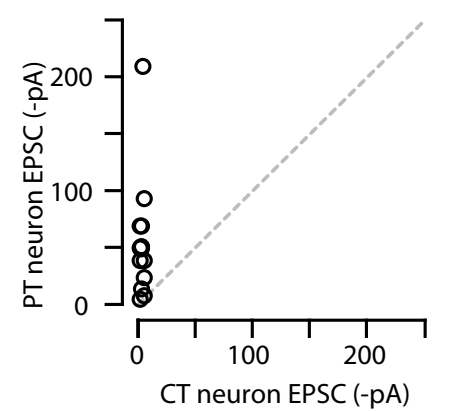

Figure 4. CT and PT neurons are mostly not interconnected. $\boldsymbol{A}$, Epifluorescence image of CT neurons labeled with ChR2 in M1 near the injection site. Coronal section of Ntsr1-Cre mouse. Scale bar, $0.5 \mathrm{~mm}$. B, Example traces of photo-evoked spikes recorded in cell-attached voltage-clamp mode from Ntsr1-ChR2 CT neurons at two different stimulus durations ( 0.2 and $5 \mathrm{~ms}$ ). Arrow marks the 0.2 ms stimulus. $C$, Average plot showing spike number (black line with scale on the left) and $P_{\text {spike }}$ (gray line with scale on the right) obtained at different photostimulation widths. Arrow marks the stimulus duration that produced similar responses to RV-ChR2 CT neurons. D, Schematic of stimulation/recording configuration. $\boldsymbol{E}$, Example traces of EPSCs recorded from CT and PT neurons upon photostimulation of RV-ChR2 (top) or Ntsr1-ChR2 (bottom) CT neuron population. $\boldsymbol{F}$, Pairwise comparison of CT and PT responses to RV-ChR2 (filled circles) and Ntsr1-ChR2 (open circles) CT stimulation. G, Epifluorescence image of RV-ChR2expressing PT neurons. Scale bar, $0.5 \mathrm{~mm}$. $\boldsymbol{H}$, Schematic of stimulation/recording configuration. I, Example traces of EPSCs recorded from CT and PT neurons upon photostimulation of PT neuron population. $\boldsymbol{J}$, PT input to CT versus PT neurons, as in $\boldsymbol{F}$.

Disynaptic inhibition scales with excitation in a pathway-specific manner In these studies of intracortical excitatory connections formed by CT neurons with IT and PT neurons, the recording conditions allowed sampling of the disynaptic inhibitory responses in the same cells by setting the command potential to $0 \mathrm{mV}$, the reversal potential for excitatory responses (Fig. 3F). Photostimulation of CT neurons evoked IPSCs in CT and IT neurons, but generally only for IT neurons located in layer 6, not 5B (Fig. 5A). Therefore, similar to $\mathrm{CT} \rightarrow \mathrm{IT}$ excitatory connections, CT $\rightarrow$ interneuron $\rightarrow$ IT connections were also layer dependent; IT-5B neurons received almost no inhibition and IT-6 neurons received similar inhibition compared with CT neurons (median ratio of $\mathrm{CT} \rightarrow$ interneuron $\rightarrow$ IT-5B/CT $\rightarrow$ interneuron $\rightarrow \mathrm{CT}: 0.03 ; \quad p=0.002$, sign test, 10 pairs; median ratio of $\mathrm{CT} \rightarrow$ interneuron $\rightarrow$ IT-6/CT $\rightarrow$ interneuron $\rightarrow$ CT: $0.66 ; p=0.07$, sign test, 14 pairs; Fig. 5A).

In the reverse direction (i.e., IT $\rightarrow$ interneuron $\rightarrow$ CT or IT), inhibitory responses were robust and of similar amplitude in CT and IT neurons (median ratio of $\mathrm{IT} \rightarrow$ interneuron $\rightarrow \mathrm{CT} / \mathrm{IT} \rightarrow$ interneuron $\rightarrow$ IT-5B: $0.72 ; p=0.45$, sign test, 7 pairs, 5 slices; median ratio of IT $\rightarrow$ interneuron $\rightarrow \mathrm{CT} / \quad$ IT $\rightarrow$ interneuron $\rightarrow$ IT-6: $0.68 ; p=0.45$, sign test, 7 pairs; Fig. 5B).

Analysis of disynaptic inhibition between CT and PT neurons showed that photostimulation of CT neurons generated $\sim 15$-fold less inhibition in postsynaptic PT compared with CT neurons (median ratio of $\mathrm{CT} \rightarrow$ interneuron $\rightarrow \mathrm{PT} /$ $\mathrm{CT} \rightarrow$ interneuron $\rightarrow \mathrm{CT}: 0.13 ; p=0.13$, sign test, 4 pairs for RV-ChR2; and 0.16; $p=0.18$, sign test, 9 pairs for Ntsr1-ChR2; $0.16, p=0.02$, for pooled RV-ChR2 and Ntsr1-ChR2 data; Fig. 5C). In the reverse direction $(\mathrm{PT} \rightarrow$ interneuron $\rightarrow \mathrm{CT}$ or $\mathrm{PT})$, the IPSCs showed a similarly asymmetric pattern as the EPSCs, with relatively sign test, 11 pairs, 3 animals, 5 slices for Ntsr1-ChR2) (Fig. 4D$F)$. Therefore, overall, these data indicate $\mathrm{CT} \rightarrow \mathrm{PT}$ connectivity is relatively rare and often essentially absent.

Next, we tested connectivity in the reverse direction, from PT to CT and PT. For this, we returned to the use of RV to label PT neurons with ChR2 (again, injecting RV-ChR2 into the contralateral cervical spinal cord; Fig. 4G). This experiment showed virtually no input to CT neurons from $\mathrm{PT}$ neurons (median ratio of $\mathrm{PT} \rightarrow \mathrm{CT} / \mathrm{PT} \rightarrow \mathrm{PT}: 0.01 ; p=0.0002$, sign test, 13 pairs, 4 animals, 6 slices; Fig. $4 H-J)$.

Together, these data indicated that these two classes of M1 projection neurons each formed within-class connections (CT $\leftrightarrow$ $\mathrm{CT}$ and $\mathrm{PT} \leftrightarrow \mathrm{PT})$, but their across-class connectivity $(\mathrm{CT} \leftrightarrow \mathrm{PT})$ was largely absent; that is, they were mostly unconnected. strong $\mathrm{PT} \rightarrow$ interneuron $\rightarrow \mathrm{PT}$ responses, but no $\mathrm{PT} \rightarrow$ interneuron $\rightarrow$ CT responses (median ratio of $\mathrm{PT} \rightarrow$ interneuron $\rightarrow \mathrm{PT} /$ $\mathrm{PT} \rightarrow$ interneuron $\rightarrow \mathrm{CT}: 0.00, p=0.0002$, sign test, 13 pairs; Fig. 5D).

These results indicate that disynaptic inhibition was engaged in a systematic manner, with multiple determinants of specificity. First, for all neurons from all experiments in which both excitatory (E) and inhibitory (I) responses were sampled, I scaled with E. An example of such a scaling obtained from three neurons recorded in one slice is shown in Figure $5 E$. Therefore, I/E ratios were generally (indeed, remarkably) constant, albeit with variability across individual cells (Fig. $5 F$ ). Second, this variability was partly due to pathway-specific differences in I/E ratios, which were higher after photostimulation of CT neurons compared 

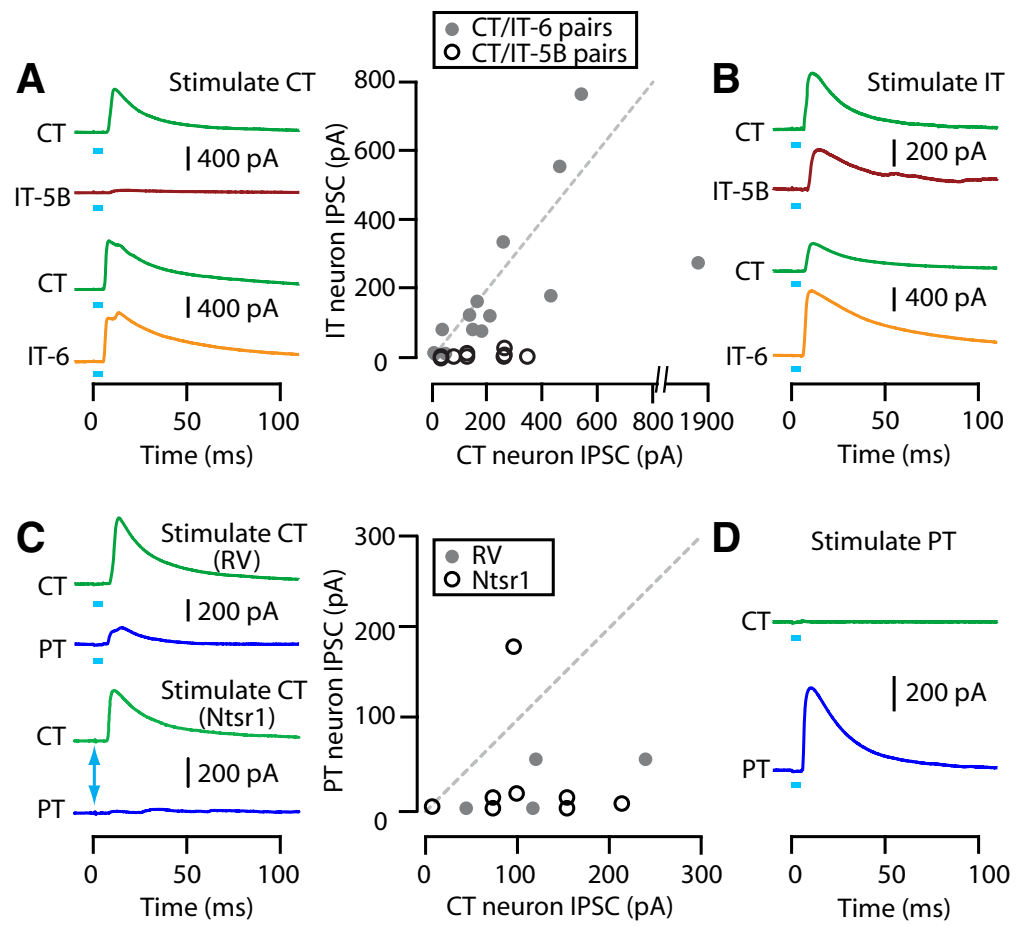

\section{$\mathbf{E}$}

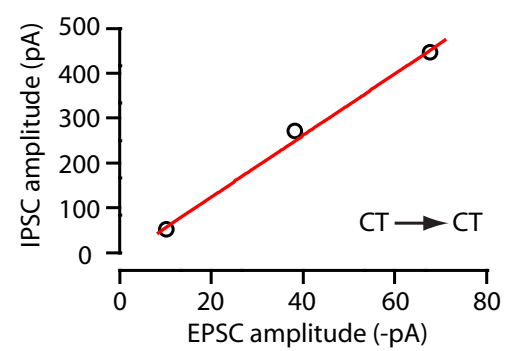

$\mathbf{F}$

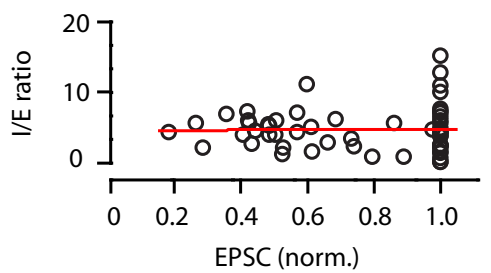

G

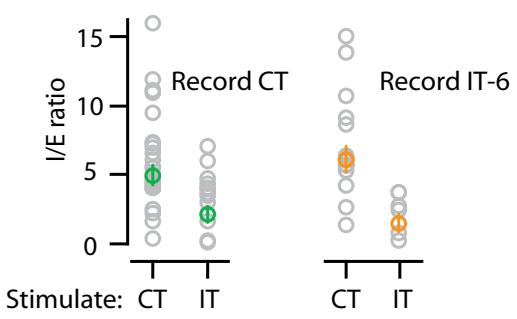

H

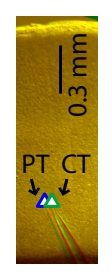

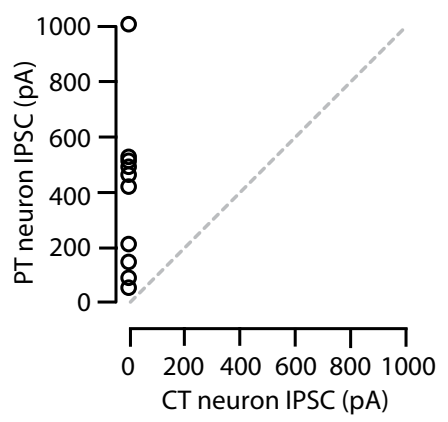

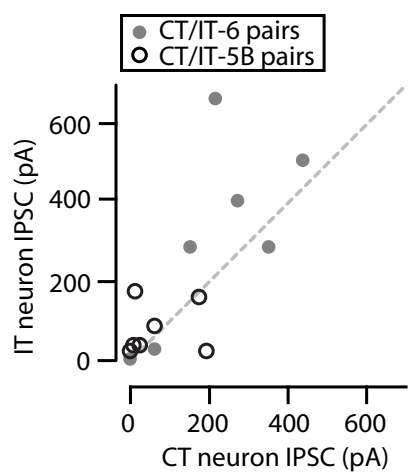

Figure 5. Disynaptic inhibition (I) parallels excitatory input (E) and I/E ratios are pathway specific. $A$, Example traces (left) and pairwise comparison of IPSCS recorded from CT and IT-5B (middle) or CT and IT-6 (right) neurons upon photostimulation of CT neurons. B, Plot represented as in $\boldsymbol{A}$, but for IT neuron stimulation. $\boldsymbol{C}$, Example traces (left) and pairwise comparison of IPSCS recorded from CT and PT neurons upon photostimulation of CT neurons, for RV-ChR2 (top) and Ntsr1-ChR2 (bottom) photostimulation. Arrow marks the 0.2 ms stimulus used for Ntsr1-ChR2. D, Traces and plot represented as in $\boldsymbol{C}$, but for PT neuron stimulation. $\boldsymbol{E}$, Representative plot of EPSC versus IPSC amplitudes for three neurons recorded in the same slice in the RV-ChR2 experiments. Line: linear regression $\left(R^{2}=0.99\right)$. $\boldsymbol{F}$, Ratio of IPSC/EPSC plotted against normalized EPSC amplitude per slice. Data points with EPSC amplitudes $<15 \mathrm{pA}$ were excluded. Line indicates linear regression $\left(R^{2}=\right.$ 0.0003). G, Comparison of I/E ratio for CT (left) or IT-6 (right) neurons when stimulating different presynaptic populations (CT or IT). $\boldsymbol{H}$, Recordings from a neighboring pair of CT and PT neurons. The PT neuron received strong PT excitation and disynaptic inhibition; the CT neuron received neither.

with IT neurons (I/E ratio for $\mathrm{CT} \rightarrow \mathrm{CT}$ and $\mathrm{IT} \rightarrow \mathrm{CT}$ pathways: $4.9 \pm 0.7$ and $2.1 \pm 0.6, p=0.01$, rank-sum test, $n=24$ and 13 neurons; I/E ratio for CT $\rightarrow$ IT- 6 and IT $\rightarrow$ IT -6 pathways: $6.1 \pm$ 0.9 and $1.5 \pm 0.6, p=0.003$, rank-sum test, $n=16$ and 6 neurons; Fig. $5 G$ ). Third, this scaling of I in proportion to $\mathrm{E}$ was observed even in the extreme for adjacent pairs of neurons in which one neuron received strong $\mathrm{E}$ and I input and the other received neither E nor I input. In the example shown (Fig. $5 H$ ), photostimulation of PT neurons evoked strong E and I in a PT neuron, but essentially zero E and I in a neighboring CT neuron, even though these neurons were separated by only $\sim 75 \mu \mathrm{m}$. Therefore, from these experiments, we conclude that disynaptic inhibition in these CT-related microcircuits is both postsynaptically cell specific, in that $\mathrm{E}$ and I remain proportional ('balanced') on a cell-by-cell basis according to the precise level of excitation received by individual postsynaptic neurons, and presynaptically class specific, in that the proportionality constant (ratio of I to E) varies according to the presynaptic (but not postsynaptic) projection class.

\section{TC axons innervate IT and PT neurons strongly but CT neurons only weakly}

Having assessed the intracortical circuit organization of M1-CT neurons, we next addressed their connections with thalamic neurons. TC neurons in VL innervate neurons in layers $5 \mathrm{~A}$ and $5 \mathrm{~B}$ in vibrissal motor cortex, including PT neurons (Hooks et al., 
A

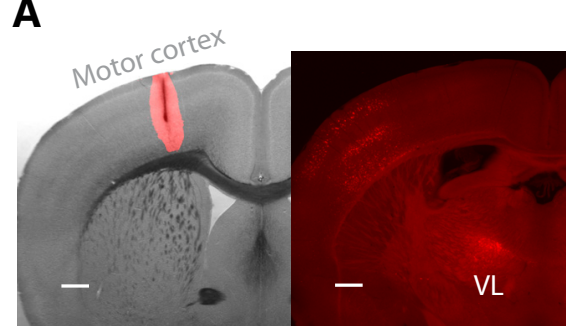

B

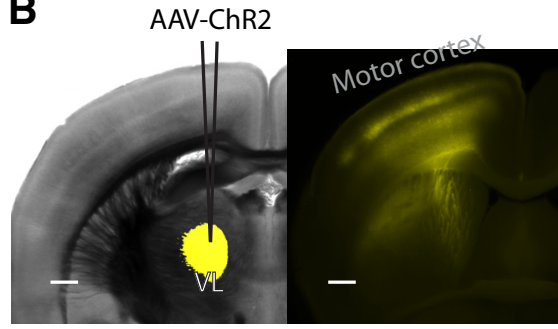

C

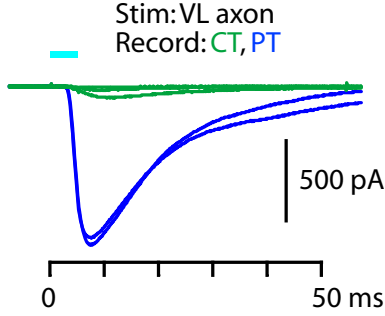

D

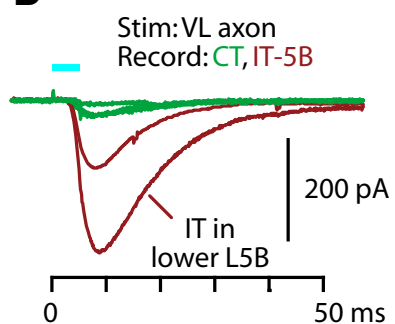

E
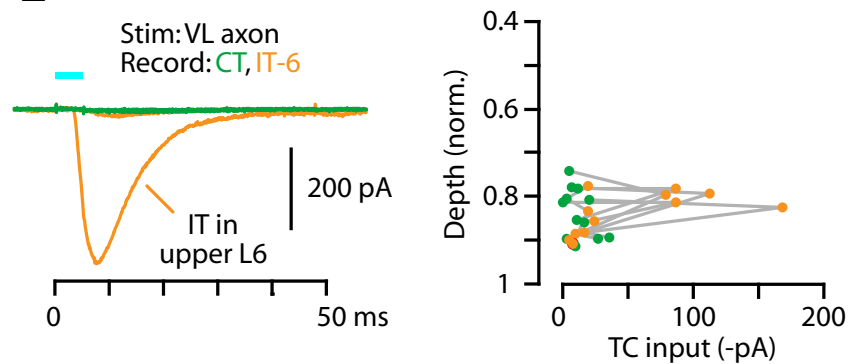
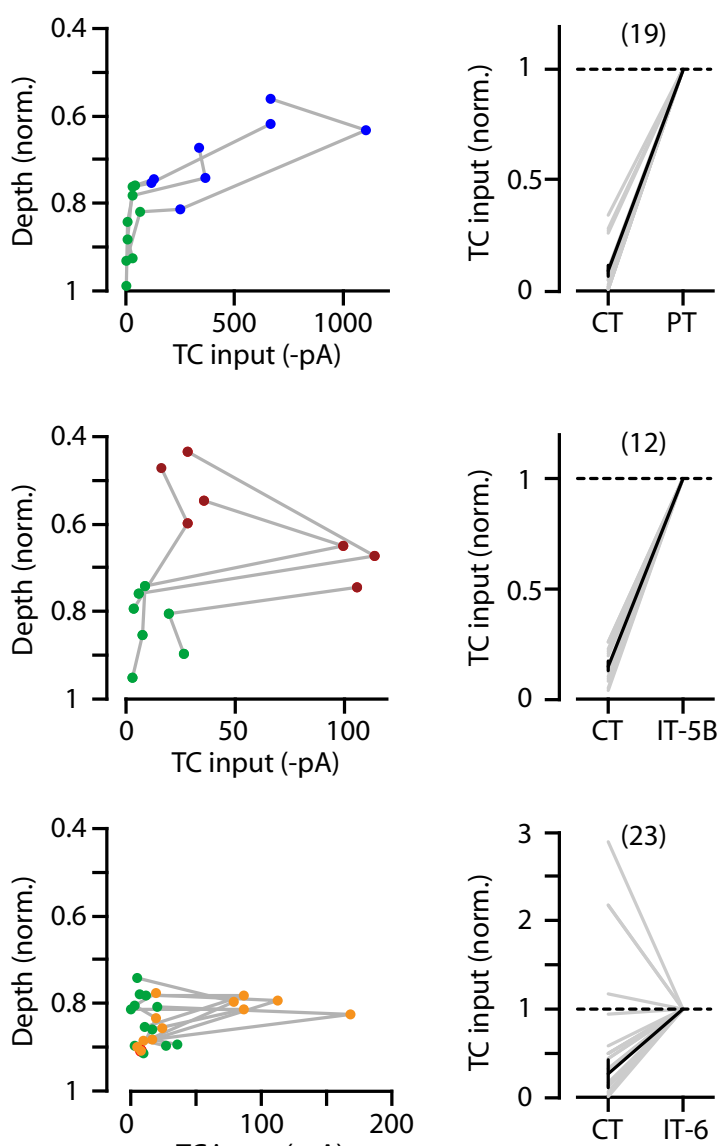

$\mathbf{F}$

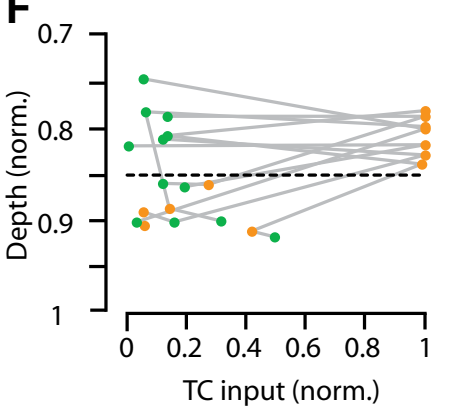

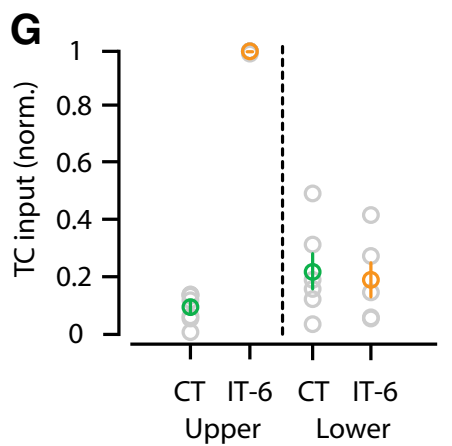

Figure 6. TC axons innervate primarily PT and IT, not CT. $\boldsymbol{A}$, Left, Bright-field image of coronal slice containing M1 with superimposed epifluorescence image of retrograde tracer injection site (red). Right, Epifluorescence image of resulting M1p-TC neuron labeling in thalamus. Scale bar, $0.5 \mathrm{~mm}$. B, Left, Bright-field image of coronal section containing VL with superimposed epifluorescence image of AAV-ChR2 injection site (yellow). Right, Epifluorescence image of resulting labeling pattern of TC axons in M1. Scale bar, $0.5 \mathrm{~mm}$. C, Left: Example traces of EPSCs recorded from CT (green) and PT (blue) neurons (in same slice). Middle, TC input profile for CT and PT neurons as a function of normalized soma depth from pia. Lines connect data points from the same slice. Right, Normalized pairwise comparison of TC input to CT and PT neurons (geometric mean \pm SEM). D, Same as in C but for CT (green) and IT-5B (dark red) neurons. E, Same as in Cbut for CT (green) and IT-6 (orange) neurons. $\boldsymbol{F}$, Same plot as in $\boldsymbol{E}$ (middle) but with data normalized to the strongest-responding neuron per slice. Dashed line separates upper and lower regions of layer 6. G, Plots comparing TC input with upper and lower CT and IT-6 neurons.
2013), but their relative targeting of different projection neurons (CT, IT, PT) in layer $5 \mathrm{~B}$ and 6 has not been fully resolved, particularly in the forelimb M1 area studied here. Injection of retrograde tracer into M1 resulted in labeling of M1projecting thalamocortical (M1p-TC) neurons in multiple thalamic nuclei, especially VL (Tlamsa and Brumberg, 2010; Hooks et al., 2013; Fig. 6A). The photoexcitability of ChR2-expressing axons in slices permits analysis of long-range inputs (Petreanu et al., 2007). We therefore injected AAV-ChR2 into VL and prepared M1 slices. The resulting multibanded labeling pattern of TC axons in M1 (Fig. 6B) was similar to that previously observed in vibrissal motor cortex (Hooks et al., 2013). We recorded from identified projection neurons in these M1 slices and measured the photo-evoked TC input. In this set of experiments, TTX $(1 \mu \mathrm{M})$ and 4-AP $(100 \mu \mathrm{M})$ were added to the bath to isolate just the monosynaptic excitatory inputs (Petreanu et al., 2009), eliminating the theoretical possibility of polysynaptic activity via intracortical circuits (e.g., $\mathrm{TC} \rightarrow \mathrm{IT} \rightarrow \mathrm{CT}$, based on the results presented above).

CT neurons received vastly less TC input compared with either PT (median ratio of TC $\rightarrow$ CT/TC $\rightarrow$ PT: $0.05, p<10^{-5}$, sign test, 19 pairs, 4 slices, 2 animals; Fig. $6 C$ ) or IT-5B (median ratio of TC $\rightarrow$ CT/ TC $\rightarrow$ IT -5B: $0.16, p=0.0005$, sign test, 12 pairs, 4 slices, 2 animals; Fig. $6 D$ ) neurons. TC input was furthermore significantly weaker onto CT than to neighboring IT- 6 neurons (median ratio of $\mathrm{TC} \rightarrow \mathrm{CT} /$ TC $\rightarrow$ IT-6: $0.33 ; p=0.003$, sign test, 23 pairs, 6 slices, 4 animals; Fig. $6 E$ ). However, strong TC input to IT- 6 neurons was only observed for neurons located in the upper part of layer 6 (Fig. $6 F, G$ ).

Collectively, these data indicate a pattern of relatively strong excitation by TC input from VL to PT neurons and to IT neurons located in layer $5 \mathrm{~B}$ and upper layer 6, with only weak excitation of CT and lower IT-6 neurons.

\section{CT axons only weakly excite TC} neurons in VL, but strongly excite thalamic neurons in several other nuclei

Many studies have shown that, anatomically, M1 projects densely to VL and several other thalamic nuclei, including VM, PO, and RTN (Terashima et al., 1987; Aldes, 1988; Cicirata et al., 1990; Deschênes et al., 1994; Hoffer and Alloway, 2001; Kakei et al., 2001; Puelles et al., 2012; Hooks et al., 2013; Oh et al., 2014; Fig. 

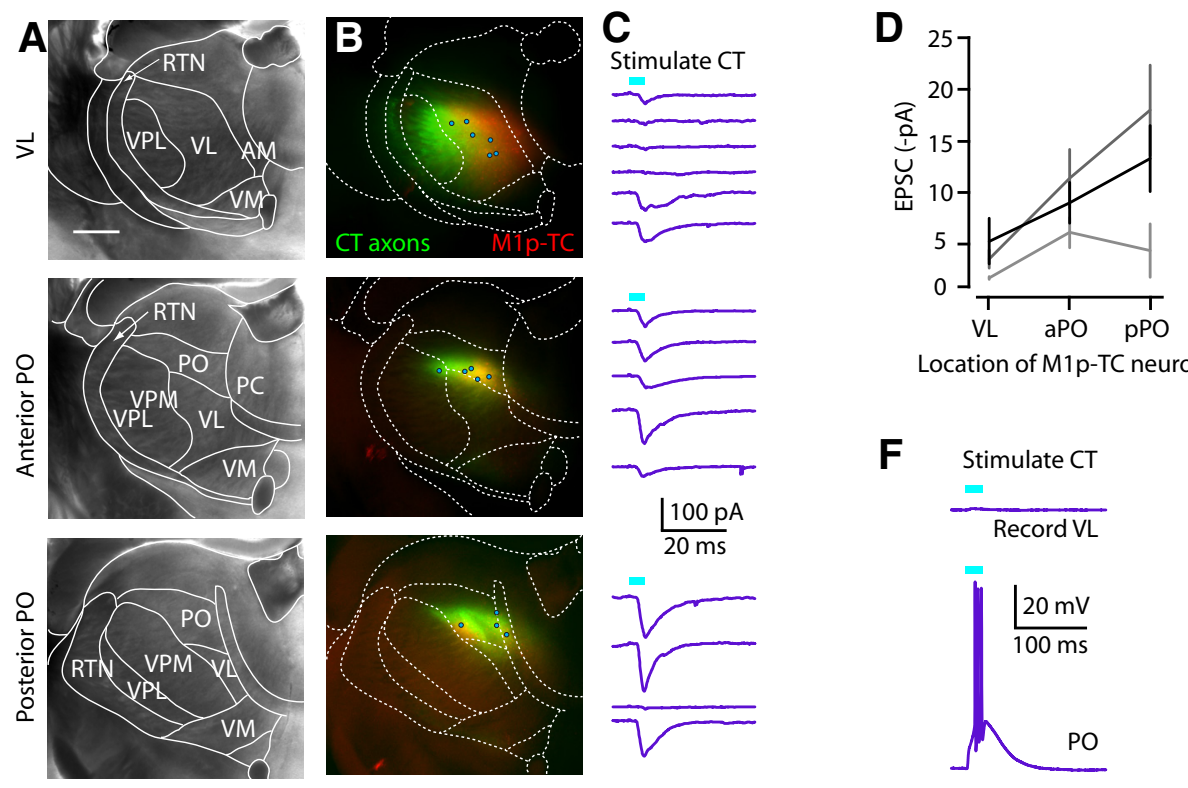

Location of M1p-TC neurons
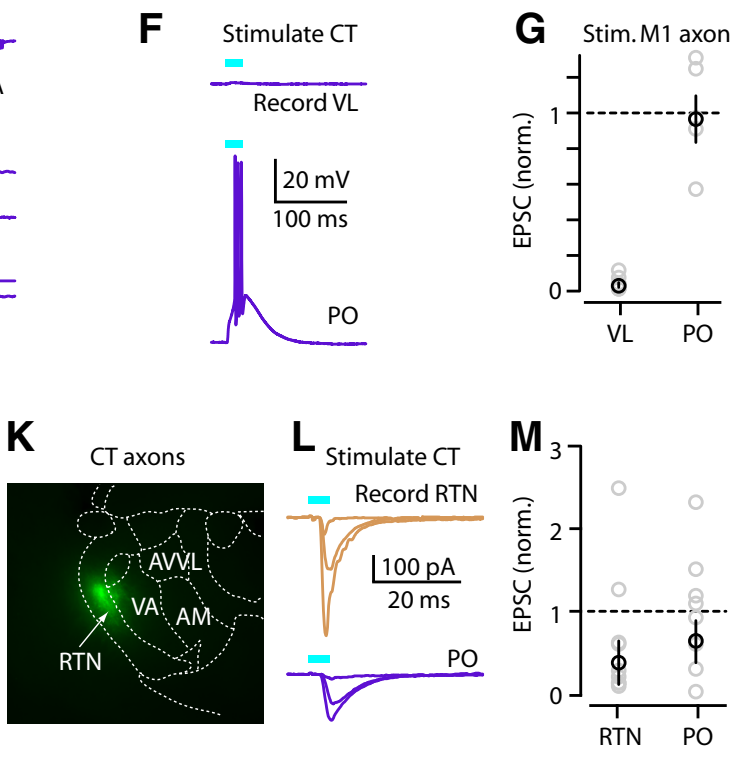

Figure 7. CT axons only weakly excite TC neurons in VL, but strongly excite thalamic neurons in several other nuclei. $\boldsymbol{A}$, Bright-field image of coronal section containing VL (top), aPO (middle), and pP0 (bottom). Borders of thalamic nuclei were determined as described in Figure 1. Scale bar, $0.5 \mathrm{~mm}$. $\boldsymbol{B}$, Merged epifluorescence image of CT axons (green) and M1p-TC neurons (red) in the same slices. Circles represent the locations where M1P-TC neurons were recorded in the same slices (total of 15 neurons in this animal). $C$, EPSC traces from the recorded M1p-TC neurons. Traces in each panel are ordered lateral to medial. Each line represents the set of multiple single-cell recordings obtained from one animal. $\boldsymbol{D}, \mathrm{CT}$ input to M1p-TC neurons in VL and anterior and posterior P0. $\boldsymbol{E}$, Normalized plot of CT input to VL and P0. aP0 and pP0 data were pooled. Individual EPSCs were divided with mean input to P0. $F$, Current-clamp traces from M1p-TC neuron in VL and P0 of same animal. CT input could evoke spikes in P0 but not in VL. $\boldsymbol{G}$, Same plot as $\boldsymbol{E}$, but for M1 input to M1p and unlabeled TC neurons. $\boldsymbol{H}$, EPSC traces from multiple M1p-TC neurons in VM and P0 of same animal. I, Normalized plot of CT input to M1p-TC neurons in VM and P0. Values were normalized to mean input to PO. J, Normalized plot of CT input to M1p-TC neurons in VL and VM. Values were normalized to mean input to VM. $\boldsymbol{K}$, Epifluorescence image of coronal section containing RTN and CT axons (green). $\boldsymbol{L}$, EPSC traces obtained from multiple neurons in RTN and P0 of one animal. $\boldsymbol{M}$, Normalized pairwise comparison of CT input to neurons in RTN versus M1p-TC neurons in P0.

$1 A, B)$, but synaptic connections to thalamic neurons have not been evaluated electrophysiologically in this system. Of particular interest are M1-CT inputs to M1p-TC neurons because these would constitute monosynaptic closure of the motor cortico-thalamo-cortical loop. To investigate this, we coinjected AAV-flex-ChR2 and a retrograde tracer (CTB647) into M1 of Ntsr1-Cre mice to label CT axons and M1p-TC somata in thalamus, respectively. In VL, the density of CT axons was highest laterally, whereas the density of M1p-TC neurons was highest medially (Fig. $7 A, B$, top row); this pattern of opposing gradients of CT axons and M1p-TC neurons within VL was consistently observed across experiments ( $n=8$ animals). We conducted electrophysiological recordings from M1p-TC neurons in VL, in this case without TTX and 4-AP in the bath (both to increase sensitivity for detecting inputs and because polysynaptic excitation was not a concern in thalamus).

Recording from M1p-TC neurons in VL showed responses to CT stimulation that were mostly weak and typically undetectable, even for laterally located neurons for which the overlap with CT axons was greatest (Fig. $7 C$, top right). Indeed, the examples shown include the strongest responses observed in any of these experiments.

In contrast, we observed strong spatial overlap between M1-CT axon labeling and M1p-TC neurons in a posterior region identified as the PO (Paxinos and Franklin, 2001; Dong, 2008; Puelles et al., 2012; Hooks et al., 2013), which receives excitatory inputs from PT neurons in somatosensory cortex and from ascending paralemniscal pathways (Groh et al., 2014). The PO extended over the two slices immediately posterior to that containing VL (Fig. 7B, middle and bottom rows). Recordings from M1p-TC neurons in both its anterior and posterior portions (aPO and pPO) showed strong excitatory input from M1-CT neurons, for most PO neurons (Fig. $7 C$, middle and bottom rows). Because results for aPO and $\mathrm{pPO}$ were similar $(0.48 \pm 0.1$ vs $0.64 \pm 0.22$, normalized to the mean value for $\mathrm{pPO}, p=0.15$, signed-rank test, $n=14$ and 11 neurons, 3 animals, 3 slices each; Fig. 7D), we pooled them for analysis. We quantified the relative amount of CT input to M1p-TC neurons in VL and PO for slices from the same animals $(n=3)$. On average, CT input to M1p-TC neurons was only $\sim 20 \%$ in VL compared with PO $(\mathrm{CT} \rightarrow \mathrm{VL}$ vs 
A
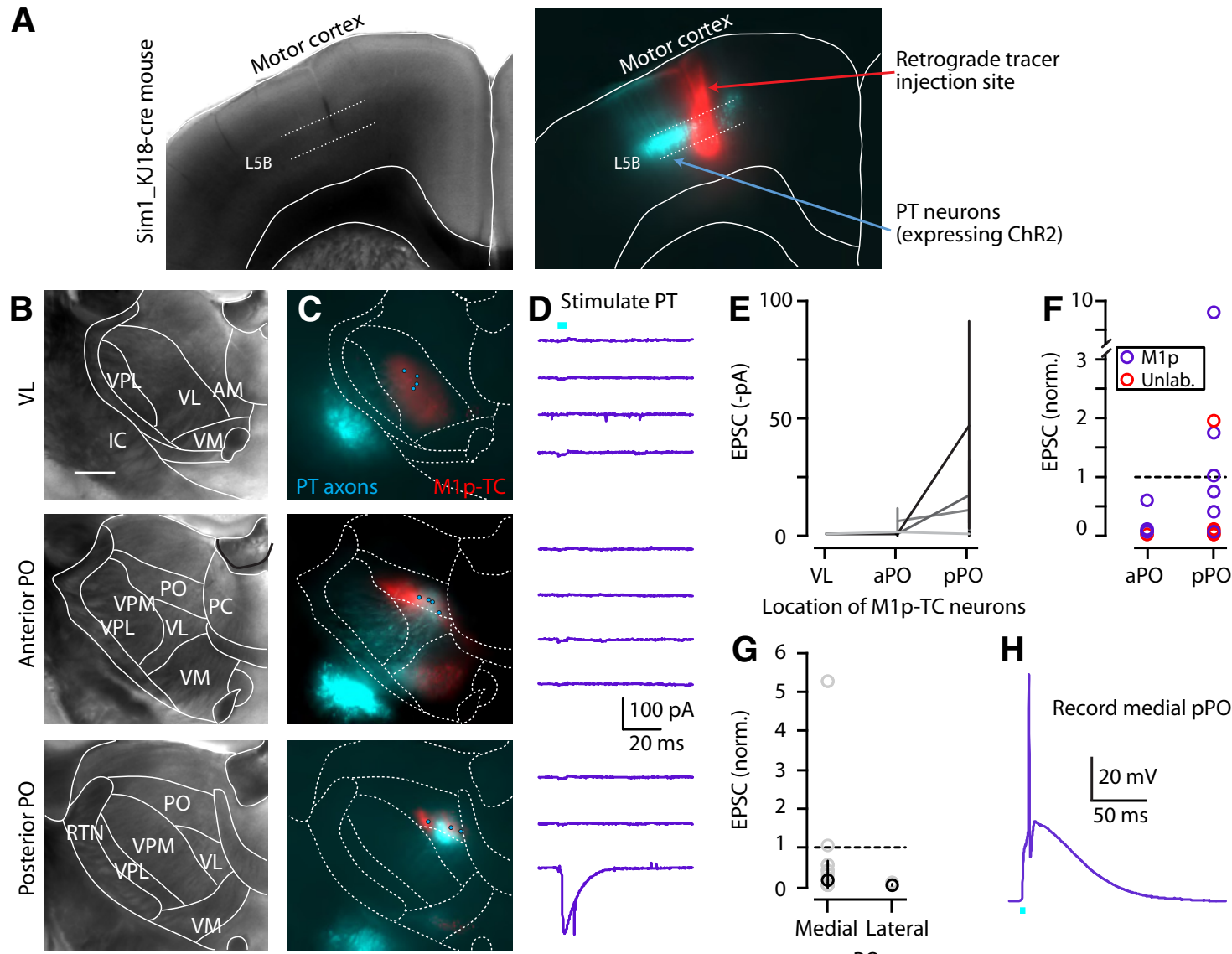

Location of M1p-TC neurons

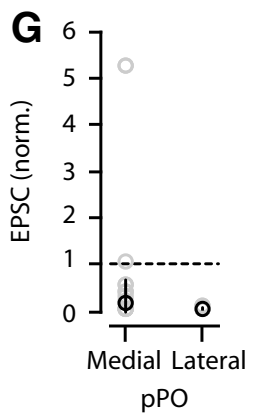

H

Record medial pPO

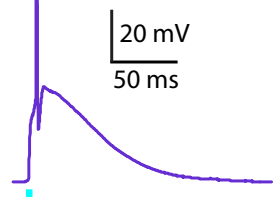

Figure 8. PT axons excite TC neurons in medial portion of pPO.A, Bright-field (left) and epifluorescence (right) images of a coronal section showing M1 of Sim1_KJ18-Cre mice, with PT neurons (cyan) labeled by injection of Cre-dependent AAV-ChR2 virus injection and also the retrograde tracer injection site (red) in M1 to label TC neurons in the thalamus. $\boldsymbol{B}$, Bright-field image of coronal section containing VL (top), aPO (middle), and pPO (bottom) of Sim1_KJ18-Cre mice injected with AAV-ChR2 as in $\boldsymbol{A}$. Borders of thalamic nuclei were determined as described in Figure 1. Scale bar, $0.5 \mathrm{~mm}$. C, Merged epifluorescence image of PT axons (cyan) and M1p-TC neurons (red) in the same slices. Circles represent the locations where thalamic neurons were recorded in the same slices (total of 11 neurons in this animal). D, EPSC traces from the recorded thalamic neurons including M1p-TC and unlabeled neurons. Traces in each panel are ordered lateral to medial. Each line represents the set of multiple single-cell recordings obtained from one animal. $E$, PT input to M1p-TC neurons in VL and anterior and posterior P0. Note a lack of PT input in VL. $\boldsymbol{F}$, Normalized plot of PT input to aPO and pPO. Individual EPSCs were divided with mean input to PPO. G, Normalized plot of PT input to thalamic neurons in medial and lateral cluster of M1p-TC neurons in pPO. Individual EPSCs were divided with mean input to PPO. Plot includes unlabeled thalamic neurons. $\boldsymbol{H}$, Current-clamp traces from M1p-TC neuron in medial pPO. PT input could evoke spikes in this region.

$\mathrm{CT} \rightarrow \mathrm{PO}: 0.13 \pm 0.09$ vs $0.71 \pm 0.15, p=0.003$, signed-rank test, $n=16$ and 25 neurons, 3 animals, 3 slices each; Fig. $7 D, E)$. Indeed, in current-clamp recordings, $\mathrm{CT}$ input was sufficient to evoke action potentials in some M1p-TC neurons in PO but not VL (Fig. $7 F$ ).

To confirm this unexpectedly weak CT input to VL, we conducted the same type of experiment, but used wild-type animals and injected M1 with AAV-ChR2 and retrograde tracer (CTB647) to label both CT and PT axons projecting to thalamus with ChR2. Responses again showed weak-to-absent input to VL $(\mathrm{M} 1 \rightarrow \mathrm{VL}$ vs $\mathrm{M} 1 \rightarrow \mathrm{PO}: 0.02 \pm 0.01$ vs $0.96 \pm 0.13, n=12$ and 6 neurons, $p=0.03$, signed-rank test, 2 animals, 2 slices each; Fig. $7 G$ ). The relatively weak input to VL neurons was not limited to M1p-TC neurons, because unlabeled neurons located in the midst of the densest CT axon labeling also received weak input $(0.00 \pm 0.00, n=8)$. Therefore, neither CT nor PT axons provided strong input to either labeled M1p-TC or unlabeled TC neurons in VL.

CT axons and M1p-TC neurons were also observed in VM, which receives afferents from the basal ganglia (Herkenham, 1979; Puelles et al., 2012). Recording from M1p-TC neurons in VM showed relatively strong input from CT axons that was not significantly different from the strong CT inputs to PO neurons, but was significantly stronger than the weak inputs to VL neurons observed in the same animals $(\mathrm{CT} \rightarrow \mathrm{VM}$ vs $\mathrm{CT} \rightarrow \mathrm{PO}: 0.21 \pm 0.24$ vs $0.76 \pm 0.32 \mathrm{pA} ; n=8$ and 9 neurons, $p=0.25$; signed-rank test, 3 animals, 3 slices; $\mathrm{CT} \rightarrow \mathrm{VL}$ vs $\mathrm{CT} \rightarrow \mathrm{VM}: 0.02 \pm 0.01$ vs $0.58 \pm 0.51 \mathrm{pA}, n=6$ and 8 neurons $p=0.03,2$ animals, 2 slices each; Fig. $7 \mathrm{H}-J)$.

Last, we investigated the connectivity of CT axons to neurons in the RTN, where CT axon labeling was also observed. Recordings from RTN neurons showed mostly strong responses, comparable to those observed in $\mathrm{PO}(\mathrm{CT} \rightarrow \mathrm{RTN}$ vs $\mathrm{CT} \rightarrow \mathrm{PO}: 0.38 \pm$ 0.26 vs $0.65 \pm 0.25, \mathrm{pA} ; n=9$ and 8 neurons, $p=0.39$, signedrank test, 3 animals, 4 slices for RTN and 3 slices for PO; Fig. $7 K-M)$.

From these results, we conclude that CT innervation is strongest for M1p-TC neurons in PO, followed by VM and RTN, and notably weak-to-absent for VL neurons.

\section{PO neurons are also excited by PT axons from M1}

The preferential targeting of M1-CT neurons to PO over VL raises the question of how M1-PT neurons connect onto neurons in these thalamic nuclei. To address this, we injected AAV-flexChR2 and retrograde tracer (CTB647) into M1 of a recently characterized PT-specific mouse line (Sim1_KJ18-Cre line; 
Gerfen et al., 2013) and subsequently prepared thalamic slices (Fig. 8A). Among the VL, aPO, and pPO slices prepared from these mice (identified based on location of M1p-TC neuron clusters, as in Fig. 7A,B), M1-PT axons were labeled only weakly in VL (Fig. $8 B, C$ ) in all animals $(n=6)$, and recordings from M1p-TC or non-M1p-TC neurons in VL (using the same conditions as in the preceding CT$\rightarrow$ thalamus experiments) indicated essentially zero PT input to these neurons $(0.59 \pm 0.12 \mathrm{pA}, n=8$ neurons, 3 animals, 3 slices; Fig. $8 D, E$ ), confirming our finding of generally weak $\mathrm{M} 1 \rightarrow \mathrm{VL}$ connectivity (Fig. 7). In contrast, PT axons were observed both in aPO and pPO slices, but localized to the medial portion of the cluster of M1p-TC neurons. Electrophysiological recordings from thalamic neurons in these areas (either from M1p-TC or non-M1p-TC neurons) showed the strongest responses in $\mathrm{pPO}$ $(\mathrm{PT} \rightarrow \mathrm{aPO}$ vs $\mathrm{PT} \rightarrow \mathrm{pPO}: 0.03 \pm 0.04$ vs $0.10 \pm 0.60, p=0.02$, signed-rank test, $n=8$ and 16 neurons, 3 animals, 3 slices each; Fig. 8F). However, there was large variability in $\mathrm{PT}$ input among $\mathrm{pPO}$ neurons, with only those (either M1p-TC or non-M1p-TC) located in medial M1p-TC clusters receiving PT input (medial pPO vs lateral pPO: $0.16 \pm 0.49$ vs $0.01 \pm 0.01, p=0.001$, signed-rank test, 14 pairs, 3 animals, 3 slices each; Fig. $8 G$ ). For some PO neurons, $\mathrm{PT}$ input was sufficiently strong to evoke action potentials (Fig. $8 H$ ). These data indicate that the axons of both CT and PT neurons target thalamic neurons in a similar distribution of nuclei (weak to VL, strong to PO). Together, these observations strongly suggest that TC neurons in the medial subregion of $\mathrm{pPO}$ integrate inputs from CT and PT neurons in M1.

\section{Discussion}

\section{Intracortical circuits of M1-CT neurons}

The intracortical connections of M1-CT neurons were highly specific and equally notable for the connections present and absent. Excitatory input to CT neurons came from CT and IT neurons, but not PT neurons. IT input was additionally layer specific, arising from IT neurons primarily in deeper but not upper layers. Excitatory output from CT neurons went to CT and IT neurons, but connections to PT neurons were scarce. The innervation of IT neurons was additionally layer specific, restricted to those in layer 6 , not layer 5B.

When combined with previous evidence for mostly unidirectional IT $\rightarrow$ PT connectivity in M1 (Kiritani et al., 2012), a picture emerges of a highly specific intracortical circuit enabling fairly extensive direct communication between CT and IT neurons, but not between CT and PT neurons (Fig. 9A). The striking paucity of CT-PT interconnectivity implies that these two projection classes can function relatively independently of each other. This arrangement is consistent with in vivo recordings in $\mathrm{M} 1$ of high $\mathrm{PT}$ but mostly low CT activity during motor behavior (Beloozerova et al., 2003a; Beloozerova et al., 2003b; Sirota et al., 2005); indeed, it provides a circuit-level mechanism whereby the activities of these two classes of M1 output neurons may be uncoupled. Unitary connections between PT and CT neurons have been reported thalamus.
B

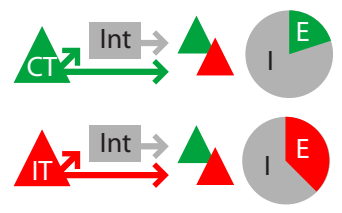

C
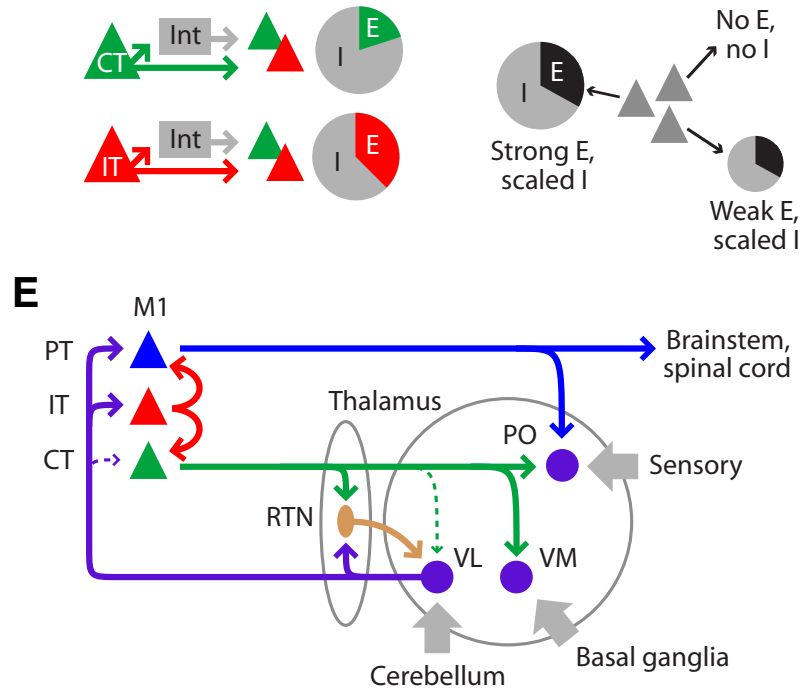

Cerebellum

Figure 9. Schematic diagrams of intracortical and thalamo-cortico-thalamic circuits of M1-CT neurons. $\boldsymbol{A}$, Projection-specific connectivity among $\mathrm{CT}$, IT, and PT neurons. $\boldsymbol{B}$, I/E ratios were presynaptically specific, being higher for CT than for IT neurons. Int, erneurons. $C$, In individual postsynaptic neurons (of any projection class; gray pyramids), disynaptic inhibition scaled in amplirons. In extreme, neighboring neurons receiving no excitation received no disynaptic inhibition either (Fig. 5H). D, Innervation of PT and IT but not CT neurons by TC axons from VL. E, Summary of thalamo-cortico-thalamic circuit organization between M1 and

in sensory areas but may be infrequent (Thomson, 2010). The intralaminar CT-IT connectivity observed here is broadly consistent with expectations from previous studies in sensory cortex, showing CT $\leftrightarrow$ IT connectivity in layer 6 , including a similar bias toward stronger $\mathrm{CT} \rightarrow \mathrm{CT}$ than $\mathrm{CT} \rightarrow \mathrm{IT}$ connections (Mercer et al., 2005; Thomson, 2010). The topographic pattern of local excitatory inputs to CT neurons arising primarily from deeper layers and not upper layers resembles that described for putative CT neurons in rat visual cortex (Zarrinpar and Callaway, 2006). Layer 4 neurons project prominently to CT neurons in some cortical areas and species (Briggs and Callaway, 2001; Douglas and Martin, 2004; Thomson, 2010; Tanaka et al., 2011), but this was not a strong projection for the M1-CT neurons studied here. Similarly, projections from CT to layer 5A neurons have been described in mouse primary somatosensory cortex (S1) (Kim et al., 2014), but in mouse M1, the ascending projections from layer 6 are generally weak compared with sensory areas (Weiler et al., 2008; Hooks et al., 2011).

Disynaptic inhibition systematically accompanied excitation in these pathways. Inhibitory responses recorded in any particular postsynaptic neuron scaled in amplitude with the excitatory responses in the same neuron. That is, inhibition 'balanced' excitation on a cell-by-cell basis, similar to recent findings in layer $2 / 3$ of visual cortex (Xue et al., 2014). Strikingly, the inhibitionexcitation proportionality held even in the extreme: neurons receiving no excitation also received no inhibition even when neighboring neurons received strong excitation and inhibition. A second finding was that the ratio of inhibition to excitation was dependent on which projection class provided the presynaptic excitation. In particular, I/E ratios were higher for CT than IT stimulation, a finding that is consistent with prior observations of strong $\mathrm{CT} \rightarrow$ interneuron connectivity in sensory areas (White and Keller, 1987; Mercer et al., 2005; West et al., 2006; Thomson, 2010; Olsen et al., 2012; Bortone et al., 2014). In summary, inhibition in these deeper-layer circuits was highly specific and was 
determined by both presynaptic and postsynaptic factors (Fig. $9 B, C)$. Furthermore, CT neurons were particularly effective at recruiting disynaptic inhibition in these intracortical circuits.

\section{Thalamo-cortico-thalamic circuits of M1-CT neurons}

The connections formed by M1-CT neurons with thalamic neurons were also highly specific. Regarding the connections of TC neurons to CT and other M1 neurons, the main finding was that VL inputs to CT neurons were strikingly weak, in contrast to the robust VL input to PT neurons and to IT neurons in layer $5 \mathrm{~B}$ and upper layer 6 (Fig. 9D). The lack of TC $\rightarrow$ CT connectivity was unexpected from prior studies of CT neurons in sensory cortex that have suggested direct TC innervation of CT neurons (for review, see Thomson, 2010). However, a recent study of TC projections to CT neurons in visual cortex demonstrated that ultrastructurally confirmed $\mathrm{TC} \rightarrow \mathrm{CT}$ synapses were much less abundant than expected on the basis of axon-dendrite overlap, leading to the suggestion that excitation from intracortical rather than TC sources determines CT firing activity (da Costa and Martin, 2009). Our findings support this concept, indicating that CT neurons receive the weakest TC input among the three major projection classes, but do receive local excitation from IT neurons; they likely also receive long-range cortical input from areas such as orbital cortex (Hooks et al., 2013). These observations thus carry significant implications for how information can (and cannot) flow in these thalamo-cortico-thalamic pathways through $\mathrm{M} 1$, in particular implying an obligatory polysynaptic route via IT neurons (i.e., $\mathrm{TC} \rightarrow \mathrm{IT} \rightarrow \mathrm{CT}$ ) rather than monosynaptic closure of these loops via CT neurons (i.e., $\mathrm{TC} \rightarrow \mathrm{CT}$ ).

Regarding the outputs of CT neurons to thalamus, the main finding was that VL receives the weakest input from M1-CT neurons compared with thalamic neurons in several other nuclei. This was unexpected because anatomical studies in primary sensory areas have shown that CT neurons project back to the primary sensory nucleus that innervates that cortical area; for example, CT neurons project from $\mathrm{S} 1$ to the ventrobasal nucleus and from primary visual cortex to the lateral geniculate nucleus (Thomson, 2010; Sherman, 2012). Here, although M1-CT axons projected anatomically to VL, the electrophysiologically assayed connections were mostly weak to absent. In contrast, M1-CT input was stronger to neurons in the reticular nucleus (RTN) and TC neurons in the VM nucleus, and strongest to TC neurons in the PO nucleus, which is usually considered a higher-order sensory nucleus. Cortical innervation of higher-order thalamic nuclei is usually associated with PT neurons (Reichova and Sherman, 2004; Sherman, 2012), which do not innervate RTN, and, consistent with this idea, we found inputs from M1-PT neurons to PO neurons, particularly in medial PO. Prior anatomical studies in rodent S1 have demonstrated that some CT neurons also project to higher-order areas such as PO (Zhang and Deschênes, 1997). The weak M1-CT input to VL neurons could reflect modulatory properties of CT outputs (Reichova and Sherman, 2004). We did not explore whether sustained presynaptic stimulation might generate facilitating responses characteristic of modulatory connections (Sherman, 2012). However, CT axons did provide strong (driver-like) excitation to other thalamic neurons, especially in $\mathrm{PO}$, where they evoked action potentials.

This $\mathrm{M} 1 \rightarrow \mathrm{PO}$ projection characterized here carries several functional implications. PO neurons have been shown to integrate driver-like inputs from both $\mathrm{PT}$ neurons in primary somatosensory cortex and from brainstem sources (Groh et al., 2014). Our results indicate that CT and PT neurons in M1 provide two additional sources of driver-like inputs, at least to a subset of PO neurons (whether these two pathways converge at the level of single PO neurons was not investigated here). These $\mathrm{CT} \rightarrow \mathrm{PO}$ and $\mathrm{PT} \rightarrow \mathrm{PO}$ pathways could potentially be independently regulated because $\mathrm{CT}$ and $\mathrm{PT}$ neurons were found to be unconnected (discussed above). Furthermore, these two pathways could function in parallel with a polysynaptic M1 $\rightarrow$ PO disinhibitory circuit via zona incerta (Urbain and Deschênes, 2007). These results support the idea of $\mathrm{PO}$ as a key node in the sensorimotor network involved in active sensation closely linked with motor as well as somatosensory cortical areas.

Because both $\mathrm{TC} \rightarrow \mathrm{CT}$ connections within cortex and $\mathrm{CT} \rightarrow \mathrm{TC}$ connections within VL were weak to absent, our findings lead to the surprising conclusion that motor cortex and motor thalamus are not directly linked through monosynaptic TC $\leftrightarrow$ CT loops. Furthermore, because RTN neurons inhibit TC neurons, this arrangement suggests that M1-CT neurons exert primarily an inhibitory influence over TC neurons in VL (i.e., $\mathrm{CT} \rightarrow \mathrm{RTN} \rightarrow \mathrm{TC}$ ). In contrast, sensory CT neurons exert a mixed excitatory-inhibitory influence in primary sensory thalamus (Cruikshank et al., 2010; Olsen et al., 2012). The functional implications of this arrangement remain to be investigated, but could potentially include a reduction in the sensitivity of TC neurons in VL to cerebello-thalamic excitatory inputs and/or an enhancement of the tuning properties of TC neurons, similar to recent in vivo findings in sensory systems (Temereanca and Simons, 2004; Olsen et al., 2012; Mease et al., 2014). Whereas the synaptic circuits of M1-CT neurons appear to be organized to promote primarily inhibition in VL, they appear to be organized to promote primarily excitation in $\mathrm{PO}$ and $\mathrm{VM}$, implying that activity in M1-CT neurons may enhance the transfer of sensoryand basal-ganglia-related activity to M1 while suppressing cerebello-thalamo-cortical activity. Of course, our measurements of static connectivity leave open the possibility that dynamic properties of synaptic connections could modulate signaling in these circuits in vivo; however, the low firing rates of M1-CT neurons in vivo (discussed above) imply that these neurons may operate to a large extent in a low-frequency regime with the static-like connectivity characterized here. Clearly, many questions remain about these selectively connected, curiously inactive, yet numerically abundant neurons and their roles in motor behavior. Collectively, the findings reported here provide a detailed new circuit-level framework (Fig. 9E) for further investigating the functions of M1-CT neurons.

\section{References}

Aldes LD (1988) Thalamic connectivity of rat somatic motor cortex. Brain Res Bull 20:333-348. CrossRef Medline

Anderson CT, Sheets PL, Kiritani T, Shepherd GMG (2010) Sublayerspecific microcircuits of corticospinal and corticostriatal neurons in motor cortex. Nat Neurosci 13:739-744. CrossRef Medline

Apicella AJ, Wickersham IR, Seung HS, Shepherd GMG (2012) Laminarly orthogonal excitation of fast spiking and low threshold spiking interneurons in mouse motor cortex. J Neurosci 32:7021-7033. CrossRef Medline

Beloozerova IN, Sirota MG, Swadlow HA (2003a) Activity of different classes of neurons of the motor cortex during locomotion. J Neurosci 23:1087-1097. Medline

Beloozerova IN, Sirota MG, Swadlow HA, Orlovsky GN, Popova LB, Deliagina TG (2003b) Activity of different classes of neurons of the motor cortex during postural corrections. J Neurosci 23:7844-7853. Medline

Bortone DS, Olsen SR, Scanziani M (2014) Translaminar inhibitory cells recruited by layer 6 corticothalamic neurons suppress visual cortex. Neuron 82:474-485. CrossRef Medline

Briggs F, Callaway EM (2001) Layer-specific input to distinct cell types in layer 6 of monkey primary visual cortex. J Neurosci 21:3600-3608. Medline

Cicirata F, Angaut P, Serapide MF, Panto MR (1990) Functional organiza- 
tion of the direct and indirect projection via the reticularis thalami nuclear complex from the motor cortex to the thalamic nucleus ventralis lateralis. Exp Brain Res 79:325-337. CrossRef Medline

Cruikshank SJ, Urabe H, Nurmikko AV, Connors BW (2010) Pathwayspecific feedforward circuits between thalamus and neocortex revealed by selective optical stimulation of axons. Neuron 65:230-245. CrossRef Medline

da Costa NM, Martin KA (2009) Selective targeting of the dendrites of corticothalamic cells by thalamic afferents in area 17 of the cat. J Neurosci 29:13919-13928. CrossRef Medline

Deschênes M, Bourassa J, Pinault D (1994) Corticothalamic projections from layer $\mathrm{V}$ cells in rat are collaterals of long-range corticofugal axons. Brain Res 664:215-219. CrossRef Medline

Dong HW (2008) The Allen Reference Atlas. Hoboken, NY: Wiley.

Douglas RJ, Martin KA (2004) Neuronal circuits of the neocortex. Annu Rev Neurosci 27:419-451. CrossRef Medline

Gerfen CR, Paletzki R, Heintz N (2013) GENSAT BAC cre-recombinase driver lines to study the functional organization of cerebral cortical and basal ganglia circuits. Neuron 80:1368-1383. CrossRef Medline

Ginger M, Haberl M, Conzelmann KK, Schwarz MK, Frick A (2013) Revealing the secrets of neuronal circuits with recombinant rabies virus technology. Front Neural Circuits 7:2. CrossRef Medline

Gong S, Doughty M, Harbaugh CR, Cummins A, Hatten ME, Heintz N, Gerfen CR (2007) Targeting Cre recombinase to specific neuron populations with bacterial artificial chromosome constructs. J Neurosci 27: 9817-9823. CrossRef Medline

Groh A, Bokor H, Mease RA, Plattner VM, Hangya B, Stroh A, Deschênes M, Acsády L (2013) Convergence of cortical and sensory driver inputs on single thalamocortical cells. Cereb Cortex 24:3167-3179. CrossRef Medline

Hattox AM, Nelson SB (2007) Layer V neurons in mouse cortex projecting to different targets have distinct physiological properties. J Neurophysiol 98:3330-3340. CrossRef Medline

Herkenham M (1979) The afferent and efferent connections of the ventromedial thalamic nucleus in the rat. J Comp Neurol 183:487-517. CrossRef Medline

Hoffer ZS, Alloway KD (2001) Organization of corticostriatal projections from the vibrissal representations in the primary motor and somatosensory cortical areas of rodents. J Comp Neurol 439:87-103. CrossRef Medline

Hooks BM, Hires SA, Zhang YX, Huber D, Petreanu L, Svoboda K, Shepherd GMG (2011) Laminar analysis of excitatory local circuits in vibrissal motor and sensory cortical areas. PLoS Biol 9:e1000572. CrossRef Medline

Hooks BM, Mao T, Gutnisky DA, Yamawaki N, Svoboda K, Shepherd GMG (2013) Organization of cortical and thalamic input to pyramidal neurons in mouse motor cortex. J Neurosci 33:748-760. CrossRef Medline

Hunnicutt BJ, Long BR, Kusefoglu D, Gertz KJ, Zhong H, Mao T (2014) A comprehensive thalamocortical projection map at the mesoscopic level. Nat Neurosci 17:1276-1285. CrossRef Medline

Kakei S, Na J, Shinoda Y (2001) Thalamic terminal morphology and distribution of single corticothalamic axons originating from layers 5 and 6 of the cat motor cortex. J Comp Neurol 437:170-185. CrossRef Medline

Kim J, Matney CJ, Blankenship A, Hestrin S, Brown SP (2014) Layer 6 corticothalamic neurons activate a cortical output layer, layer 5 a. J Neurosci 34:9656-9664. CrossRef Medline

Kiritani T, Wickersham IR, Seung HS, Shepherd GMG (2012) Hierarchical connectivity and connection-specific dynamics in the corticospinalcorticostriatal microcircuit in mouse motor cortex. J Neurosci 32:49925001. CrossRef Medline

Kita T, Kita H (2012) The subthalamic nucleus is one of multiple innervation sites for long-range corticofugal axons: a single-axon tracing study in the rat. J Neurosci 32:5990-5999. CrossRef Medline

Kumar P, Ohana O (2008) Inter- and intralaminar subcircuits of excitatory and inhibitory neurons in layer 6a of the rat barrel cortex. J Neurophysiol 100:1909-1922. CrossRef Medline

Kuramoto E, Furuta T, Nakamura KC, Unzai T, Hioki H, Kaneko T (2009) Two types of thalamocortical projections from the motor thalamic nuclei of the rat: a single neuron-tracing study using viral vectors. Cereb Cortex 19:2065-2077. CrossRef Medline

Landisman CE, Connors BW (2007) VPM and PoM nuclei of the rat so- matosensory thalamus: intrinsic neuronal properties and corticothalamic feedback. Cereb Cortex 17:2853-2865. CrossRef Medline

Lee SC, Cruikshank SJ, Connors BW (2010) Electrical and chemical synapses between relay neurons in developing thalamus. J Physiol 588:24032415. CrossRef Medline

Mease RA, Krieger P, Groh A (2014) Cortical control of adaptation and sensory relay mode in the thalamus. Proc Natl Acad Sci U S A 111:6798 6803. CrossRef Medline

Mercer A, West DC, Morris OT, Kirchhecker S, Kerkhoff JE, Thomson AM (2005) Excitatory connections made by presynaptic cortico-cortical pyramidal cells in layer 6 of the neocortex. Cereb Cortex 15:1485-1496. CrossRef Medline

Oh SW, Harris JA, Ng L, Winslow B, Cain N, Mihalas S, Wang Q, Lau C, Kuan L, Henry AM, Mortrud MT, Ouellette B, Nguyen TN, Sorensen SA, Slaughterbeck CR, Wakeman W, Li Y, Feng D, Ho A, Nicholas E, et al. (2014) A mesoscale connectome of the mouse brain. Nature 508:207214. CrossRef Medline

Olsen SR, Bortone DS, Adesnik H, Scanziani M (2012) Gain control by layer six in cortical circuits of vision. Nature 483:47-52. CrossRef Medline

Osakada F, Mori T, Cetin AH, Marshel JH, Virgen B, Callaway EM (2011) New rabies virus variants for monitoring and manipulating activity and gene expression in defined neural circuits. Neuron 71:617-631. CrossRef Medline

Parent M, Parent A (2006) Single-axon tracing study of corticostriatal projections arising from primary motor cortex in primates. J Comp Neurol 496:202-213. CrossRef Medline

Paxinos G, Franklin KBJ (2001) The mouse brain in stereotaxic coordinates, Ed 2. London: Academic.

Petreanu L, Huber D, Sobczyk A, Svoboda K (2007) Channelrhodopsin-2assisted circuit mapping of long-range callosal projections. Nat Neurosci 10:663-668. CrossRef Medline

Petreanu L, Mao T, Sternson SM, Svoboda K (2009) The subcellular organization of neocortical excitatory connections. Nature 457:1142-1145. CrossRef Medline

Puelles L, Martinez-de-la-Torre M, Ferran J-L, Watson C (2012) Diencephalon. In: The mouse nervous system (Watson C, Paxinos G, eds), pp 313-336. Amsterdam: Academic.

Reichova I, Sherman SM (2004) Somatosensory corticothalamic projections: distinguishing drivers from modulators. J Neurophysiol 92:21852197. CrossRef Medline

Schubert D, Staiger JF, Cho N, Kötter R, Zilles K, Luhmann HJ (2001) Layer-specific intracolumnar and transcolumnar functional connectivity of layer V pyramidal cells in rat barrel cortex. J Neurosci 21:3580-3592. Medline

Shepherd GMG (2012) Circuit mapping by ultraviolet uncaging of glutamate. Cold Spring Harb Protoc 2012:998-1004. CrossRef Medline

Shepherd GMG (2014) Diversity and complexity in the pyramidal tract projectome. Nat Rev Neurosci 15:63. CrossRef Medline

Shepherd GMG (2013) Corticostriatal connectivity and its role in disease. Nat Rev Neurosci 14:278-291. CrossRef Medline

Shepherd GMG, Pologruto TA, Svoboda K (2003) Circuit analysis of experience-dependent plasticity in the developing rat barrel cortex. Neuron 38:277-289. CrossRef Medline

Sherman SM (2012) Thalamocortical interactions. Curr Opin Neurobiol 22:575-579. CrossRef Medline

Sirota MG, Swadlow HA, Beloozerova IN (2005) Three channels of corticothalamic communication during locomotion. J Neurosci 25:5915-5925. CrossRef Medline

Suter BA, O'Connor T, Iyer V, Petreanu LT, Hooks BM, Kiritani T, Svoboda K, Shepherd GMG (2010) Ephus: multipurpose data acquisition software for neuroscience experiments. Front Neural Circuits 4:100. CrossRef Medline

Suter BA, Migliore M, Shepherd GM (2013) Intrinsic electrophysiology of mouse corticospinal neurons: a class-specific triad of spike-related properties. Cereb Cortex 23:1965-1977. CrossRef Medline

Tanaka YR, Tanaka YH, Konno M, Fujiyama F, Sonomura T, OkamotoFuruta K, Kameda H, Hioki H, Furuta T, Nakamura KC, Kaneko T (2011) Local connections of excitatory neurons to corticothalamic neurons in the rat barrel cortex. J Neurosci 31:18223-18236. CrossRef Medline

Temereanca S, Simons DJ (2004) Functional topography of corticothalamic 
feedback enhances thalamic spatial response tuning in the somatosensory whisker/barrel system. Neuron 41:639-651. CrossRef Medline

Terashima T, Inoue K, Inoue Y, Mikoshiba K (1987) Thalamic connectivity of the primary motor cortex of normal and reeler mutant mice. J Comp Neurol 257:405-421. CrossRef Medline

Thomson AM (2010) Neocortical layer 6, a review. Front Neuroanat 4:13. CrossRef Medline

Tlamsa AP, Brumberg JC (2010) Organization and morphology of thalamocortical neurons of mouse ventral lateral thalamus. Somatosens Mot Res 27:34-43. CrossRef Medline

Urbain N, Deschênes M (2007) Motor cortex gates vibrissal responses in a thalamocortical projection pathway. Neuron 56:714-725. CrossRef Medline

Watakabe A, Takaji M, Kato S, Kobayashi K, Mizukami H, Ozawa K, Ohsawa S, Matsui R, Watanabe D, Yamamori T (2014) Simultaneous visualization of extrinsic and intrinsic axon collaterals in Golgi-like detail for mouse corticothalamic and corticocortical cells: a double viral infection method. Front Neural Circuits 8:110. CrossRef Medline

Weiler N, Wood L, Yu J, Solla SA, Shepherd GMG (2008) Top-down laminar organization of the excitatory network in motor cortex. Nat Neurosci 11:360-366. CrossRef Medline

West DC, Mercer A, Kirchhecker S, Morris OT, Thomson AM (2006) Layer 6 cortico-thalamic pyramidal cells preferentially innervate interneurons and generate facilitating EPSPs. Cereb Cortex 16:200-211. CrossRef Medline

White EL, Keller A (1987) Intrinsic circuitry involving the local axon collaterals of corticothalamic projection cells in mouse Sm1 cortex. J Comp Neurol 262:13-26. Medline

Wickersham IR, Finke S, Conzelmann KK, Callaway EM (2007) Retrograde neuronal tracing with a deletion-mutant rabies virus. Nat Methods 4:47-49. CrossRef Medline

Wickersham IR, Sullivan HA, Seung HS (2010) Production of glycoproteindeleted rabies viruses for monosynaptic tracing and high-level gene expression in neurons. Nat Protoc 5:595-606. CrossRef Medline

Wood L, Gray NW, Zhou Z, Greenberg ME, Shepherd GMG (2009) Synaptic circuit abnormalities of motor-frontal layer $2 / 3$ pyramidal neurons in an RNA interference model of methyl-CpG-binding protein 2 deficiency. J Neurosci 29:12440-12448. CrossRef Medline

Xue M, Atallah BV, Scanziani M (2014) Equalizing excitation-inhibition ratios across visual cortical neurons. Nature 511:596-600. CrossRef Medline

Zarrinpar A, Callaway EM (2006) Local connections to specific types of layer 6 neurons in the rat visual cortex. J Neurophysiol 95:1751-1761. CrossRef Medline

Zhang ZW, Deschênes M (1997) Intracortical axonal projections of lamina VI cells of the primary somatosensory cortex in the rat: a single-cell labeling study. J Neurosci 17:6365-6379. Medline 